\title{
Links with no exceptional surgeries
}

\author{
David Futer and Jessica S. Purcell*
}

\begin{abstract}
We show that if a knot admits a prime, twist-reduced diagram with at least 4 twist regions and at least 6 crossings per twist region, then every non-trivial Dehn filling of that knot is hyperbolike. A similar statement holds for links. We prove this using two arguments, one geometric and one combinatorial. The combinatorial argument further implies that every link with at least 2 twist regions and at least 6 crossings per twist region is hyperbolic and gives a lower bound for the genus of a link.
\end{abstract}

Mathematics Subject Classification (2000). 57M25, 57M5.

Keywords. Dehn surgery, Dehn filling, hyperbolic 3-manifolds, knot complements, link complements.

\section{Introduction}

Knots and links in $S^{3}$ are easiest to visualize with a projection diagram, but computing geometric or topological information directly from the diagram is often a difficult task. In the very special case of alternating knots, an alternating diagram reveals a lot of topological information, including the genus of the knot [5], [17] and bounds on the Heegaard genus of the complement [15]. For alternating knots and links, one can tell by looking at an alternating diagram whether the complement is hyperbolic [16], and if it is, compute upper and lower bounds on the volume [14]. However, few results of this sort extend beyond this special class of knots and links.

In this paper, we prove a mild diagrammatic condition that ensures the complement of a particular link is hyperbolic, and a slightly stronger one that ensures all non-trivial Dehn surgeries on the link are hyperbolike. We also use the combinatorial properties of a diagram to give a lower bound on the genus of a link. To state our results precisely, we will need a few definitions.

${ }^{*}$ The second author was partially supported by a VIGRE postdoc under NSF grant number DMS-0091946 at the University of Texas at Austin. 
1.1. Twist regions and reduced diagrams. A diagram $D(K)$ of a knot or link $K \subset S^{3}$ can be viewed as a 4-valent planar graph $G(K)$, with over-under crossing information at each vertex.

Definition 1.1. A bigon is a contractible region in the complement of $G(K)$ that has two edges in its boundary. Following Lackenby [12], [14], define a twist region of the knot or link to be a maximal string of bigons arranged end to end. A single crossing adjacent to no bigons is also a twist region. We implicitly assume throughout that the diagram is alternating in each twist region; otherwise, one can reduce the number of crossings of the diagram in the obvious way.

We are also concerned with the amount of twisting that occurs in each twist region. We will count this either in terms of crossings or in terms of full twists, where a full twist of one strand about the other corresponds to two crossings. See Figure 1 for an illustration of these definitions.

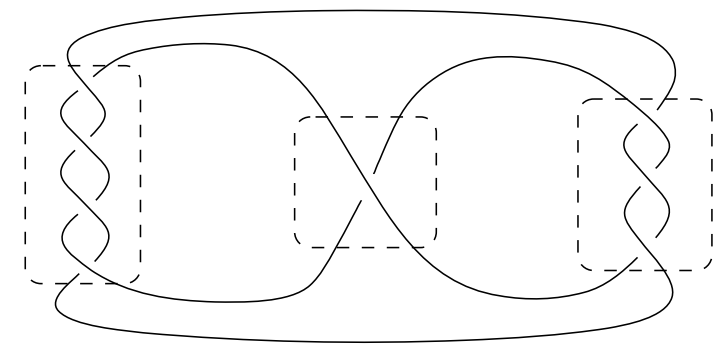

Figure 1 . The above diagram has 3 twist regions, containing $2, \frac{1}{2}$, and $1 \frac{1}{2}$ twists, respectively.

Definition 1.2. A diagram $D(K)$ of a knot or link $K \subset S^{3}$ is called prime if for any simple closed curve $\gamma$ in the projection plane that intersects the graph $G(K)$ transversely in two points in the interior of edges, $\gamma$ bounds a subdiagram containing no crossings of the original diagram. Note that this ensures the diagram contains no monogons, provided it has more than one crossing. See Figure 2.

Following Lackenby [14], we also require the diagram to be twist-reduced.

Definition 1.3. A diagram $D(K)$ of a knot or link $K$ is twist-reduced if whenever a simple closed curve $\gamma$ in the projection plane intersects the graph $G(K)$ transversely in four points in the interior of edges, with two points adjacent to one crossing and the other two points adjacent to another crossing, then $\gamma$ bounds a subdiagram consisting of a (possibly empty) collection of bigons arranged in a row between these two crossings. See Figure 2. 

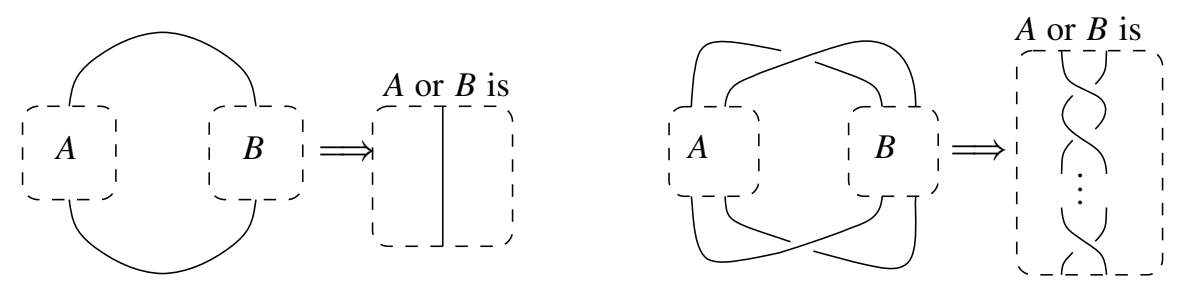

Figure 2. Left: A prime diagram; Right: A twist-reduced diagram.

Note that any diagram of a prime knot or link $K$ can be simplified into a prime, twist-reduced diagram: for if $D(K)$ is a diagram that fails to be prime, then all crossings on one side of a simple closed curve $\gamma$ are extraneous and can be removed. Similarly, if $D(K)$ is not twist-reduced, then a series of flypes will amalgamate the two twist regions adjacent to a curve $\gamma$ into a single region, reducing the number of twist regions.

These definitions allow us to state our first result.

Theorem 1.4. Let $K \subset S^{3}$ be a link with a prime, twist-reduced diagram $D(K)$. Assume that $D(K)$ has at least two twist regions (i.e. that $K$ is not a closed 2-braid). If every twist region of $D(K)$ contains at least 6 crossings, then $K$ is hyperbolic.

This result could be viewed as an extension of Menasco's theorem [16], which holds that a prime, non-split alternating link is hyperbolic whenever it is not a closed 2-braid. For alternating links, Menasco does not need any assumption on the number of crossings per twist region; to rule out non-hyperbolic links in general, some such assumption is necessary.

Recall that the genus of a link $K \subset S^{3}$ is the smallest genus of an embedded incompressible, orientable surface $S \subset S^{3}$ whose boundary is $K$. We can use prime, twist-reduced diagrams to give a lower bound on the genus.

Theorem 1.5. Let $K \subset S^{3}$ be a link of $k$ components with a prime, twist-reduced diagram $D(K)$. If $D(K)$ has $t \geq 2$ twist regions and at least 6 crossings in each twist region, then

$$
\operatorname{genus}(K) \geq\left\lceil 1+\frac{t}{6}-\frac{k}{2}\right\rceil,
$$

where $\lceil\cdot\rceil$ is the ceiling function that rounds up to the nearest integer.

Crowell [5] and Murasugi [17] have independently proved that the genus of an alternating link is equal to half the degree of its Alexander polynomial, and Gabai gave an algorithm to compute the genus of an arborescent link [9]. The advantage 
of Theorem 1.5 is that it works for general links and, in fact, gives the exact value for certain families of links.

1.2. Dehn surgery. Let $M$ be a 3-manifold with torus boundary $\partial M$, and $s$ a slope on $\partial M$, that is, $s$ is an isotopy class of essential simple closed curves on $\partial M$. The manifold obtained by gluing a solid torus $S^{1} \times D^{2}$ to $\partial M$ in such a way that the slope $s$ bounds a disk in the resulting manifold is called a Dehn filling along the slope $s$, or a Dehn surgery along $s$. More generally, if $M$ is a 3-manifold with multiple torus boundary components and along each component we have a slope $s_{i}$, we obtain a closed manifold by Dehn filling along these slopes.

Using a basis $\langle\mu, \lambda\rangle$ for the fundamental group of the torus, slopes on cusps are parameterized by $\mathbb{Q} \cup\{\infty\}$. Thus a slope corresponds to $a / b$ if and only if the slope is equivalent to $a \mu+b \lambda$. If $K$ is a knot in $S^{3}$, and $M$ is taken to be the exterior $E(K)$ of a tubular neighborhood of $K$, then we let $\mu$ correspond to a meridian, and $\lambda$ to a longitude. In this case, Dehn filling along a meridian of $K$, i.e. $1 / 0$ filling, will always give $S^{3}$. This Dehn filling is called the trivial filling. All other Dehn fillings are non-trivial.

Thurston has shown that given a hyperbolic manifold $M$ with cusps, all but finitely many choices of surgery slope on each component of $\partial M$ yield a closed hyperbolic manifold [23]. More recently, Hodgson and Kerckhoff showed that if the surgery slope on each component of $\partial M$ is longer than a given universal constant, then the resulting Dehn filled manifold is hyperbolic [11]. Using these results, Purcell was able to show that for sufficiently complicated knots, every nontrivial Dehn filling is hyperbolic [20]. However, the required knots are so complicated that they are difficult to use in practice.

If we weaken the assumption that the resulting manifold be hyperbolic, we can obtain similar surgery results for much less complicated knots.

Definition 1.6. A closed, orientable 3-manifold $M$ is hyperbolike if

(1) $M$ is irreducible and atoroidal,

(2) $M$ is not Seifert fibered, and

(3) $\pi_{1}(M)$ is infinite and word-hyperbolic.

All hyperbolic manifolds are hyperbolike. Thurston's Geometrization Conjecture [24] (whose proof was recently announced by Perelman [18], [19]) would imply the converse.

Theorem 1.7. Let $K$ be a link in $S^{3}$ with a prime, twist-reduced diagram $D(K)$. Suppose that every twist region of $D(K)$ contains at least 6 crossings and each component of $K$ passes through at least 7 twist regions (counted with multiplicity). Then every non-trivial Dehn filling of all the components of $K$ is hyperbolike. 
Notice that we obtain this information about Dehn fillings from the diagram of the link alone. We need no additional information.

The statement of Theorem 1.7 can be extended in two different ways. First, under the same hypotheses as in Theorem 1.7, a non-trivial Dehn filling of only some components of $K$ yields a hyperbolic manifold with boundary. Second, if we strengthen the hypotheses to require that each twist region contain 7 or more crossings, it will follow that a non-trivial Dehn filling of $K$ admits a negatively curved Riemannian metric. (See Theorem 3.11.) It is worth emphasizing that Perelman's recent work on the Geometrization Conjecture implies that every hyperbolike manifold and every negatively curved manifold actually admit a hyperbolic metric. However, our proofs do not rely on his papers.

Corollary 1.8. Let $K$ be a knot in $S^{3}$ with a prime, twist-reduced diagram $D(K)$. If $D(K)$ has at least 4 twist regions, and each twist region contains at least 6 crossings, then any non-trivial Dehn filling of $K$ is hyperbolike.

The corollary follows from Theorem 1.7 because if $K$ is a knot, every twist region contains two strands of $K$. Thus in a diagram with 4 twist regions, $K$ passes through a twist region 8 times.

In fact, the hypothesis of 4 twist regions in Corollary 1.8 is a sharp bound. $\mathrm{Wu}$ has shown that every pretzel knot with 3 twist regions and at least 2 crossings per twist region admits a non-trivial exceptional surgery [27]. Thus our results assume the smallest possible number of twist regions.

As for the requirement that each twist region contain at least 6 crossings, we know that some such requirement is necessary. It is known that there exist knots with nontrivial exceptional surgeries that have arbitrarily large volume, hence an arbitrarily high number of twist regions. These have been discovered by Eudave-Muñoz and Luecke [7], Eudave-Muñoz [6], as well as recently by Baker [3]. Thus a high number of twist regions alone is not enough to rule out exceptional surgeries. However, at this time the authors do not know whether the requirement of six crossings per twist region is sharp.

Another advantage of Theorem 1.7 is that it gives information on Dehn fillings without requiring us to restrict our attention to a particular class of knots or links. This should be compared to other known results. Lackenby has shown that all non-trivial Dehn surgeries on alternating knots with at least 9 twist regions are hyperbolike, as are surgeries on alternating links in which each component passes through 17 or more twist regions [12]. Wu proved that all non-trivial surgeries on a large class of arborescent knots are hyperbolic [26]. Theorem 1.7 applies to both of these classes of knots as well as non-alternating, non-arborescent knots and links.

Theorem 1.7 also gives a nice tool for understanding Dehn fillings on link complements as well as knot complements. Classifying Dehn fillings on links is often 
a more difficult problem than classifying fillings on knots, but our arguments apply equally well to both knots and links.

1.3. Two proofs, with two notions of length. By the work of Agol [2] and Lackenby [12], Dehn fillings of a hyperbolic manifold $M$ are hyperbolike whenever the surgery slopes on $\partial M$ are "sufficiently long." This term has two distinct meanings. Agol and Lackenby independently showed that if the length of each surgery slope on a maximal cusp of $M$ is at least 6, then the surgered manifold is hyperbolike. Lackenby also showed that the same conclusion holds when the combinatorial length of each surgery slope is at least $2 \pi$. In this paper, we use these two points of view to give two different proofs of Theorem 1.7.

Both proofs make use of the same surgery description of the link $K$. In Section 2, we show how to start with a prime, twist-reduced diagram of $K$ and construct a new link, whose complement in $S^{3}$ has simple geometric properties. The analysis of these properties in Section 3 leads to estimates of length on a maximal cusp, yielding our geometric proof of Theorem 1.7. In Section 4, we review relevant results from Lackenby's theory of normal and admissible surfaces in angled polyhedra, setting up the notion of combinatorial length. We then use this machinery in Section 5 to give combinatorial proofs of all three of our main theorems.

We are grateful to Henry Segerman for his helpful suggestions and to Eric Schoenfeld for providing a template for Figure 9. Robert Lipshitz has helpfully pointed out that our techniques can be used to estimate genus. Above all, we would like to acknowledge the extended guidance given to us by Steve Kerckhoff. The bulk of these results were obtained while both authors were his students.

\section{Augmented links}

In this section, we describe how to start with a prime, twist-reduced projection of a link $K$, construct a flat augmented link $L$, and subdivide the exterior $S^{3} \backslash L$ into two hyperbolic ideal polyhedra. This construction is originally due to Ian Agol and Dylan Thurston (see the appendix of [14]). We use the ideal polyhedra to find geometric information about the cusps of the complement of $L$ in $S^{3}$.

2.1. Constructing the augmented link. Let $D(K)$ be a prime, twist-reduced diagram of a link $K \subset S^{3}$. As described in the introduction, each twist region in $D(K)$ consists of two strands of $K$ wrapping around each other. For each twist region $R_{i}$, add a simple closed curve $C_{i}$ encircling the twist region, known as a crossing circle. Let $I$ be the resulting link.

Definition 2.1. For a link $K \subset S^{3}$, let the exterior $E(K)$ denote the complement of an open tubular neighborhood of $K$. 
Note that the manifold $E(I)$ is homeomorphic to the manifold $E(J)$, where $J$ is the simpler link with all full twists removed at each twist region of $I$. We can recover the original link $K$ from $J$ by performing $1 / n_{i}$ surgery on each $C_{i},\left|n_{i}\right|$ being the number of full twists we removed. Furthermore, any Dehn filling of $K$ can be viewed as a filling of $J$. The results of this paper work by analyzing the geometry and combinatorics of $S^{3} \backslash J$.

In fact, to analyze this geometry, we will make $J$ even simpler by removing all remaining single crossings from the twist regions. The resulting link $L$ has two kinds of components: knot strands coming from $K$ that lie flat in the projection plane, and crossing circles $C_{i}$ perpendicular to the projection plane. We call $L$ a flat augmented link. If some twist region $R_{i}$ had an odd number of crossings, $E(L)$ is no longer homeomorphic to $E(J)$; indeed, $J$ and $L$ can have a different number of components. We will address this issue later, in $\$ 2.3$. See Figure 3 for a visual summary of this construction.
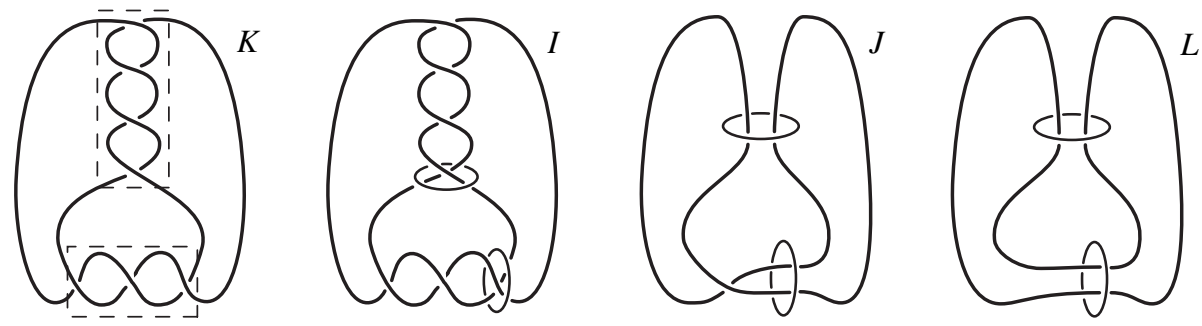

Figure 3. Left to right: The original knot with twist regions marked; the link $I$ with crossing circles added; the homeomorphic link $J$; the flat augmented link $L$.

To subdivide $S^{3} \backslash L$ into polyhedra, we first slice it along the projection plane. This divides $S^{3}$ into two identical 3-balls. Since they are identical, we focus our attention on $B_{1}$, the ball above the projection plane. The decomposition of $B_{2}$ proceeds in the same way. Each crossing circle $C_{i}$ bounds a disk $D_{i}$, half of which lies in $B_{1}$ and borders on three edges in the projection plane. We then further slice $B_{1}$ along each of these half-disks.

This allows us to pull apart the two sides of each half-disk and flatten them, creating the planar diagram of a polyhedron. (See Figure 4.) This polyhedron will inherit one face from each region of the projection diagram and one face from each side of disk $D_{i}$. We can turn this polyhedron into an ideal polyhedron $P_{1}$ by collapsing strands of $L$ to ideal vertices. The other ball $B_{2}$ becomes an identical ideal polyhedron $P_{2}$.

$P_{1}$ and $P_{2}$ will each have six edges per twist region, three from each side of the intersection between $D_{i}$ and the projection plane. At each edge, a face coming from the projection place meets a face coming from $D_{i}$. This allows us to two-color 

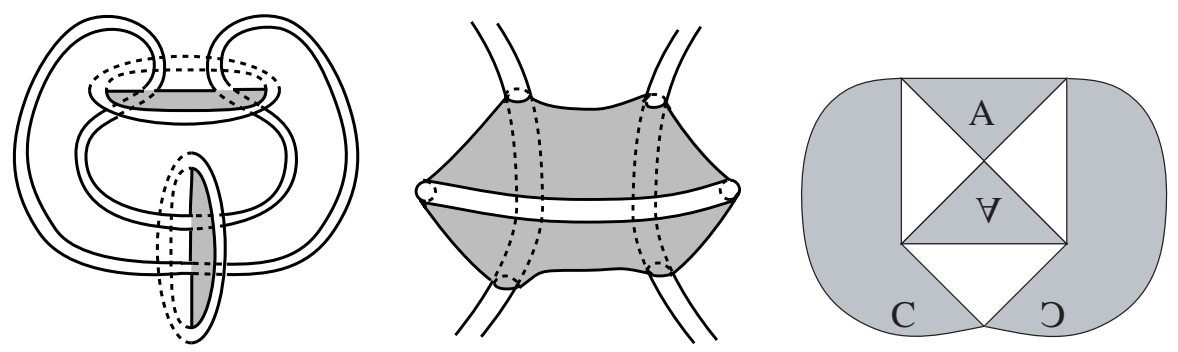

Figure 4. Decomposing $S^{3} \backslash L$ into ideal polyhedra: First slice along the projection plane, then split remaining halves of two-punctured disks. Obtain polygon on right.

the faces in a convenient fashion: the projection-plane faces will be white and the crossing-disk faces shaded, as in Figure 4.

To reconstruct $S^{3} \backslash L$ from $P_{1}$ and $P_{2}$, we first glue matching shaded faces in each $P_{j}$, and then glue the two polyhedra to each other along the white faces. Observe that in this gluing, the edges become 4-valent: each borders on two shaded faces (the two halves of $D_{i}$ ) and two white faces in the projection plane. In $\mathbb{R}^{3}$, we can position the crossing disks $D_{i}$ perpendicular to the projection plane, creating dihedral angles of $\pi / 2$ between adjacent faces. Conveniently, this feature carries over into hyperbolic geometry.

2.2. The geometry of $\boldsymbol{E}(\boldsymbol{L})$. The following theorem was used by Agol and Thurston in [14].

Theorem 2.2. Let $D(K)$ be a prime, twist-reduced diagram of a link $K$, with at least two twist regions. Let $L$ be the flat augmented link obtained from $D(K)$. Then $E(L)$ is hyperbolic. Furthermore, the polyhedra $P_{1}$ and $P_{2}$ decomposing $E(L)$ are convex ideal polyhedra in $\mathbb{H}^{3}$, with totally geodesic faces that meet at right angles.

Proof. Given the diagram $D(K)$, we can always reverse the crossings in some of the twist regions to obtain an alternating diagram $D\left(K^{\prime}\right)$. Furthermore, because the construction of the augmented link ignores the over-under crossing information, applying this construction to $K^{\prime}$ will yield the same link $L$.

Thus every flat augmented link $L$ is an example of what Adams calls an augmented alternating link. When $L$ has 2 or more crossing circles, and thus $K^{\prime}$ has 2 or more twist regions, Menasco's theorem [16] implies $E\left(K^{\prime}\right)$ is hyperbolic. Then Adams' result on augmented alternating links [1] implies that every flat augmented link $L$ is hyperbolic.

Additionally, note that there is an orientation-reversing involution of $S^{3} \backslash L$ preserving $L$ and our ideal polyhedra: namely, reflection through the projection plane. 
Every lift of this involution to the universal cover $\mathbb{H}^{3}$ is a reflection in a totally geodesic plane. Hence the polyhedra can be made totally geodesic in $\mathbb{H}^{3}$, with the shaded faces meeting the white faces at right angles.

Remark. It is worth noting that the statement and proof of Theorem 2.2 do not assume that the original link $K$ is hyperbolic. When $D(K)$ has at least two twist regions, it follows from Menasco's theorem [16] that the alternating link $K^{\prime}$ is hyperbolic; we use this to bootstrap to a hyperbolic structure on $E(L)$. This will eventually be used to prove that $K$ is hyperbolic (Theorem 1.4).

If we intersect $P_{1}$ and $P_{2}$ with the compact manifold $E(L)$, each of their ideal vertices gets truncated into a rectangular boundary face on $\partial E(L)$. If we keep track of how these rectangles are glued to one another in the gluing pattern of $P_{1}$ and $P_{2}$, we can construct a picture of the cusp triangulation of each torus of $\partial E(L)$.

Lemma 2.3. The cusp tori of $L$ are rectangular. For a crossing circle $C_{i}$, the cusp torus is composed of two boundary faces. For a knot strand $K_{j}$ lying flat in the projection plane, the cusp torus is a $2 \times n$ block of boundary faces, where $n$ is the number of twist regions crossed by $K_{j}$ (counted with multiplicity).

Proof. As we saw in the construction of $\S 2.1$, each crossing circle $C_{i} \subset L$ becomes an ideal vertex in $P_{1}$. Truncate this vertex to get a rectangular boundary face $F_{1}$. The shaded faces on opposite sides of this boundary rectangle are glued to one another in the gluing pattern, since they glue to give half the disk $D_{i}$ bounded by $C_{i}$; thus an arc in $F_{1}$ connecting the two shaded faces represents a meridian of $C_{i}$.

The two white faces meeting $F_{1}$ are glued to corresponding faces of $P_{2}$, joining $F_{1}$ to the boundary rectangle $F_{2}$. Thus the cusp torus of $C_{i}$ is tiled by $F_{1}$ and $F_{2}$, with the meridian and longitude as shown in Figure 5.
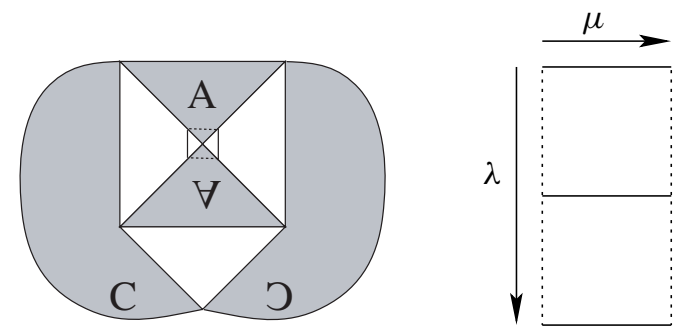

Figure 5. Left: Each crossing circle gives rise to one ideal vertex in $P_{1}$. Right: The cusp diagram corresponding to a crossing circle. Here $\mu$ is a meridian, and $\lambda$ is a longitude.

For a knot strand $K_{j} \subset L, P_{1}$ will have one ideal vertex (hence one boundary rectangle) for each strand of $K_{j}$ between adjacent crossing disks $D_{i}$. (See Figure 4.) 
These boundary rectangles are glued end to end along shaded faces coming from the $D_{i}$ to complete a longitude of $K_{j} . P_{2}$ will give rise to an identical chain of rectangles, glued to the boundary rectangles of $P_{1}$ along the white faces of the projection diagram. Thus the cusp torus of $K_{j}$ is tiled by a $2 \times n$ block of rectangles, where $n$ is the number of intersections between $K_{j}$ and the crossing disks $D_{i}$, hence equal to the number of twist regions that $K_{j}$ passes through, counted with multiplicity. See Figure 6.
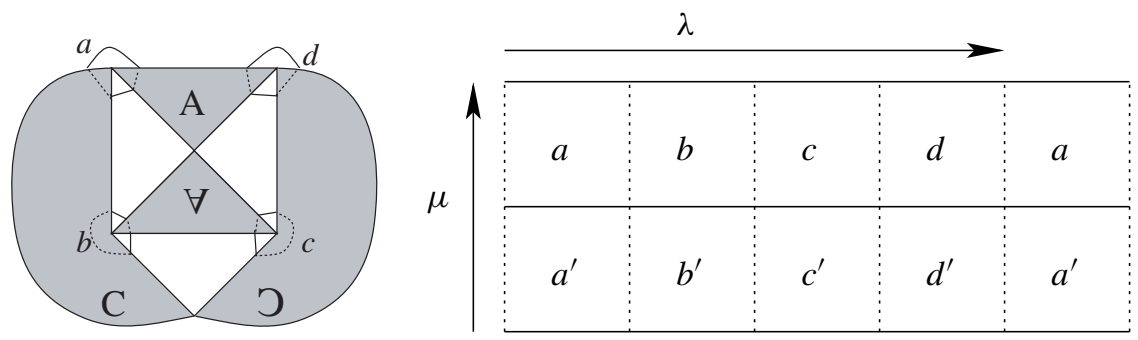

Figure 6. The cusp diagram for the knot strand cusp. Solid segments correspond to white faces, and dotted segments correspond to shaded faces.

2.3. Half-twists and surgery slopes. Recall that to construct the flat augmented link $L$ with its nice polyhedral decomposition, we took three steps, summarized in Figure 3. We added crossing circles to $K$ (obtaining a link $I$ ); removed a whole number of twists per twist region (obtaining a link $J$ with homeomorphic complement); and then removed any remaining single crossings. Whereas any Dehn filling of $K$ is a filling of $J$, the same is no longer true for $L$. Thus to obtain our results, we need to understand the combinatorics of the link $J$, with the half-twists re-inserted.

Conveniently, $E(J)$ can still be decomposed into the same polyhedra $P_{1}$ and $P_{2}$, only with a slightly modified gluing pattern. $P_{1}$ has one shaded face from each side of a crossing disk $D_{i}$; to construct $E(L)$, we glued those faces to each other. If instead we glue those shaded faces of $P_{1}$ to matching shaded faces of $P_{2}$, we effectively insert a half-twist along disk $D_{i}$ and a single crossing into the projection diagram of $L$. We can do this wherever $J$ has a single crossing. In particular, this simple rearrangement means that we have the following version of Theorem 2.2.

Theorem 2.4. Let $D(K)$ be a prime, twist reduced diagram of a link $K$. Assume that $D(K)$ has at least two twist regions, with $a_{i}$ crossings in twist region $R_{i}$. Let $J$ be the augmented link constructed in $\$ 2.1$, in which the number of crossings in region $R_{i}$ is reduced to $a_{i} \bmod 2$. Then

(1) E(J) is hyperbolic, 
(2) $E(J)$ is subdivided into convex ideal polyhedra $P_{1}$ and $P_{2}$ with dihedral angles $\pi / 2$,

(3) $K$ is the result of Dehn filling each crossing circle $C_{i}$ of $J$ along the surgery slope $1 / s_{i}$, where we removed $2\left|s_{i}\right|$ crossings from $R_{i}$, and

(4) every Dehn filling of $K$ is a filling of $J$.

Proof. The first two conclusions follow from Theorem 2.2 because $E(J)$ decomposes into the same convex ideal polyhedra as $E(L)$. The last two conclusions result from the process of constructing $J$, and are mentioned in $\$ 2.1$.

In the cusp diagrams of $\partial E(J)$, each half-twist in the transition from $L$ to $J$ will shift the gluing by one step along the shaded faces coming from $D_{i}$, as illustrated in Figure 7. Thus the neat rectangular pattern of Lemma 2.3 no longer holds. However, we can still make convenient statements about the universal cover $\widetilde{T}$ of each cusp torus of $S^{3} \backslash J$.

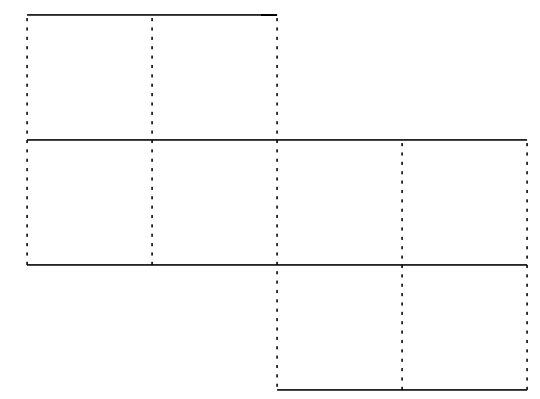

Figure 7. Cusp view: adding a half twist to a flat augmented link shifts the gluing along the shaded faces.

Definition 2.5. Let $T$ be a cusp torus of $\partial E(J)$, with universal cover $\widetilde{T}=\mathbb{R}^{2}$. Then $\widetilde{T}$ contains a rectangular lattice coming from white and shaded faces of $P_{1}$ and $P_{2}$. We construct a basis $\langle\boldsymbol{s}, \boldsymbol{w}\rangle$ of this $\mathbb{Z}^{2}$ lattice by letting $\boldsymbol{s}$ be a step parallel to a shaded face and $\boldsymbol{w}$ be a step parallel to a white face.

Lemma 2.6. Let $T$ be a cusp torus of $\partial E(J)$ and let $\langle\boldsymbol{s}, \boldsymbol{w}\rangle$ be the basis for the lattice on $\widetilde{T}$. In this basis, the fundamental domain of $T$ appears as follows:

(1) If $T$ comes from a crossing circle without a half-twist, it has meridian $w$ and longitude 2 s.

(2) If $T$ comes from a crossing circle with a half-twist, it has meridian $\boldsymbol{w} \pm \boldsymbol{s}$ (depending on the direction of the twist) and longitude $2 \mathrm{~s}$. 
(3) If $T$ comes from a component $K_{j}$ of the original link $K$, it has meridian $2 s$ and longitude $n \boldsymbol{w}+k \boldsymbol{s}$, where $K_{j}$ runs through $n$ twist regions with multiplicity and $k$ is an undetermined integer.

Proof. If $J$ does not contain any half-twists, this is a restatement of Lemma 2.3. (See Figures 5 and 6.) Each half-twist along the crossing circle $C_{i}$ shears the meridian of $C_{i}$ by $s$, as described above. It also shears by $s$ the cusp of every component of the original link $K$ passing through the crossing disk $D_{i}$.

Thus if $K_{j}$ passes through a half-twist $m$ times, the projection of the curve $n \boldsymbol{w}+m \boldsymbol{s}$ to $T$ will be some longitude of $K_{i}$, in the sense of completing a basis of $\pi_{1}(T)$ along with $\mu=2 s$. The true longitude, in the sense of having linking number 0 with $K_{j}$, is then some curve of the form $n \boldsymbol{w}+k \boldsymbol{s}$ for some integer $k$.

The basis $\langle\boldsymbol{s}, \boldsymbol{w}\rangle$ also allows us to make precise statements about the surgery curves on $\partial E(J)$ that correspond to non-trivial surgeries on $K$.

Theorem 2.7. Let $K=\bigcup_{j=1}^{m} K_{j}$ be a link in $S^{3}$ with a prime, twist reduced diagram $D(K)$. Suppose that $D(K)$ contains twist regions $R_{1}, \ldots, R_{n}(n \geq 2)$ and that twist region $R_{i}$ contains $a_{i}$ crossings. For each component $K_{j}$, let $n_{j}$ be the number of twist regions crossed by $K_{j}$, counted with multiplicity; and let $s_{j}$ be a non-trivial surgery slope on $K_{j}$.

With this notation, the surgery on $S^{3} \backslash K$ along slopes $s_{1}, \ldots, s_{m}$ can be represented as a surgery on $J$ as follows:

(1) On the (mostly) planar component of $J$ corresponding to $K_{j}$, the surgery curve is $p_{j} n_{j} w+q_{j} s$, for some integers $p_{j} \neq 0$ and $q_{j}$.

(2) On the crossing circle $C_{i}$, the surgery curve is $\boldsymbol{w} \pm a_{i} \boldsymbol{s}$.

Proof. By Lemma 2.6, $K_{j}$ has meridian $2 \boldsymbol{s}$ and a longitude of the form $n_{j} \boldsymbol{w}+k_{j} \boldsymbol{s}$. Since $s_{j}$ is a non-trivial surgery slope, it must cover at least one longitude. In particular, the number of steps that a curve representing $s_{j}$ takes along the white faces is a nonzero multiple of $n_{j}$.

To prove conclusion (2), suppose first that $a_{i}$ is even, so $J$ has no half-twist at $C_{i}$. By Lemma 2.6, $C_{i}$ has longitude $2 s$ and meridian $\boldsymbol{w}$. By Theorem 2.4, the surgery curve on $C_{i}$ traverses $a_{i} / 2$ longitudes and one meridian, proving the result.

Now, suppose that $a_{i}$ is odd. Then in the construction of $J$, we have removed $2 b_{i}=a_{i}-1$ crossings; the remaining half-twist of $J$ at $C_{i}$ goes in the same direction as the twists of $K$. By Lemma 2.6, $C_{i}$ has longitude $2 \boldsymbol{s}$ and meridian $\boldsymbol{w}+\sigma_{i} \boldsymbol{s}$, for some $\sigma_{i}= \pm 1$. By Theorem 2.4, the surgery curve traverses $\sigma_{i} b_{i}$ longitudes (with 
the same $\sigma_{i}$ ) and one meridian. Thus, in the basis of $\langle\boldsymbol{s}, \boldsymbol{w}\rangle$, the surgery curve is

$$
\begin{aligned}
\mu+\sigma_{i} b_{i} \lambda & =\left(\boldsymbol{w}+\sigma_{i} \boldsymbol{s}\right)+\sigma_{i} b_{i}(2 \boldsymbol{s}) \\
& =\boldsymbol{w}+\sigma_{i}\left(1+2 b_{i}\right) \boldsymbol{s} \\
& =\boldsymbol{w}+\sigma_{i} a_{i} \boldsymbol{s} .
\end{aligned}
$$

\section{Geometric cusp estimates}

In Section 2, we showed that each cusp of $E(J)$ contains a rectangular lattice generated by $s$ and $\boldsymbol{w}$, and in Theorem 2.7 we expressed the surgery curves on $\partial E(J)$ explicitly in terms of $s$ and $\boldsymbol{w}$. In this section, we will use the geometry of the polyhedra $P_{1}$ and $P_{2}$ to come up with lower bounds for the lengths of $s$ and $\boldsymbol{w}$ on a maximal cusp. This will allows us to estimate the lengths of surgery curves. By combining these estimates with Agol and Lackenby's 6-Theorem (Theorem 3.2), we will obtain a geometric proof of Theorem 1.7.

3.1. Length on a maximal cusp. In this paper, we measure the length of curves on a cusp of $E(J)$ in two distinct ways: geometric and combinatorial. The geometric measurements of this section come from the hyperbolic metric. A closed curve isotopic to a cusp torus has many representatives in $E(J)$, whose lengths shrink to 0 as the representative curves approach the cusp. To obtain a meaningful definition of length, we will consider curves on a horospherical torus bounding a maximal neighborhood of a cusp.

For a manifold with just one cusp, such as a knot complement, we obtain the maximal horoball neighborhood by expanding a horoball about the single cusp until it becomes tangent to itself. In a manifold with multiple cusps, such as $E(J)$, the size of the maximal cusp depends on the order in which we expand horoballs about the cusps, for a horoball might become tangent to an expanded horoball about a different cusp before it becomes tangent to itself.

Definition 3.1. Let $M$ be a 3-manifold with boundary consisting of tori, whose interior has a complete hyperbolic structure. Fix a cusp neighborhood $U$, consisting of disjoint horoball neighborhoods of the cusps of $M$. Then any closed curve $\gamma \subset \partial M$ can be assigned a unique geometric length $\ell_{g}(\gamma)$, defined as the shortest length of a curve on $\partial U$ isotopic to $\gamma$.

The subscript in $\ell_{g}(\gamma)$ serves to distinguish geometric length from the combinatorial length $\ell_{c}(\gamma)$ used in Sections 4 and 5. When the meaning is clear, we will simply use $\ell(\gamma)$.

To rule out exceptional surgeries on $M$, it helps to choose the cusp neighborhood $U$ to be maximal, to make the surgery curves as long as possible. Agol [2] and Lackenby [12] have independently proved the following surgery theorem: 
Theorem 3.2 (6-Theorem). Let $M$ be a hyperbolic 3-manifold with boundary consisting of tori. Let $s_{1}, \ldots, s_{n}$ be surgery slopes on $\partial M$, with one $s_{i}$ on each torus. Suppose that there are disjoint horoball neighborhoods of the cusps of $M$, such that $\ell_{g}\left(s_{i}\right)>6$ for all $i$. Then the manifold obtained by Dehn filling $M$ along the slopes $s_{1}, \ldots, s_{n}$ is hyperbolike.

In 33.2 , we will give explicit instructions for expanding the horoball neighborhoods about the cusps of $E(J)$ that produce favorable estimates for the length of surgery curves. These estimates will rely on lower bounds for $\ell(\boldsymbol{s})$ and $\ell(\boldsymbol{w})$.

It should be noted that the lengths of the meridians of augmented links were found independently by Eric Schoenfeld in his undergraduate thesis [22].

3.2. Horosphere packing in $\mathbb{H}^{3}$. Recall from Section 2 that each ideal vertex of $P_{1}$ and $P_{2}$ gives rise to a boundary rectangle on a cusp of $E(J)$. In the geometry of the universal cover $\mathbb{H}^{3}$, the boundary rectangle can be seen as the intersection of $P_{i}$ with a horosphere. A side parallel to $s$ is the intersection of a horosphere with a shaded face, and a side parallel to $w$ is the intersection with a white face. It turns out that boundary rectangles and horospheres are easiest to visualize in the upper half-space model of $\mathbb{H}^{3}$.

Notation. We will parameterize the upper half-space model of $\mathbb{H}^{3}$ with coordinates $(z, h)$, where $z \in \mathbb{C}$ and $h \in \mathbb{R}^{+}$. In this model, the sphere at infinity $S_{\infty}^{2}$ can be identified with the Riemann sphere $\mathbb{C} \cup \infty$.

We can apply an isometry of $\mathbb{H}^{3}$ so that the point at infinity of $\mathbb{H}^{3}$ projects to the cusp under consideration in $E(J)$. Then a horosphere about that cusp lifts to a horizontal plane at height $h$. In the metric on the upper half-space model, hyperbolic length corresponds to $\frac{1}{h}$ times the Euclidean length. Thus we can bound the hyperbolic lengths of $s$ and $\boldsymbol{w}$ using Euclidean measurements in upper half-space.

Every procedure for expanding the cusps will lower the horizontal horosphere until it becomes tangent to another horosphere. This abutting horosphere will look like a Euclidean sphere tangent to $S_{\infty}^{2}$ at some point of $\mathbb{C}$.

Definition 3.3. Let $H$ be a horosphere in the upper half-space model of $\mathbb{H}^{3}$. If $H$ is a Euclidean sphere, call the point of tangency in $\mathbb{C}$ the center of $H$. If $H$ is a horizontal Euclidean plane, we say that $H$ is centered at $\infty$.

We will normalize our horoball packing in upper half-space by placing ideal triangles into standard position.

Definition 3.4. Let $T \subset \mathbb{H}^{3}$ be an ideal triangle. We will say that $T$ is in standard position in the upper half-space model if its ideal vertices lie at 0,1 , and $\infty$. Note that any ideal triangle can be placed into standard position by an isometry. 
Recall that each shaded face of the polyhedra $P_{1}$ and $P_{2}$ is an ideal triangle. Thus we can place $P_{i}$ in the upper half-space model of $\mathbb{H}^{3}$ so that a shaded face is in standard position. When we do so, the polyhedron $P_{i}$ is lifted to lie entirely over a boundary rectangle with corners at $0,1,0+i r$ and $1+i r$ for some real number $r$.

Lemma 3.5. Arrange $P_{i}$ in $\mathbb{H}^{3}$ so that some shaded side has vertices at 0,1 , and $\infty$, so $P_{i}$ lies over a boundary rectangle with corners at $0,1,0+i r$ and $1+i r$. Follow any procedure for expanding the cusps of $E(J)$ to horoballs with disjoint interiors, and let $H$ be a horosphere centered at a point of the rectangle. If the center of $H$ does not lie on a white side of the rectangle, the diameter of $H$ is at most 1.

Proof. Since there is a shaded face of $P_{i}$ with vertices 0 and 1 , there must be a white face of $P_{i}$ containing vertices $0, i r$ and $\infty$, and another containing $1,1+i r$, and $\infty$. Recall that reflection through the white faces of $E(J)$ is an involution of the manifold, corresponding to a reflection in the projection plane of Figure 4. This involution permutes the horospheres covering cusps of $E(J)$, and thus takes $H$ either to itself or to a disjoint horosphere $H^{\prime}$. If the center of $H$ lies on a white side of the rectangle, reflection in the plane above that side will fix $H$. Otherwise, $H$ must be disjoint from its reflection. Then, since the boundary rectangle has length 1 , the diameter of $H$ can be at most 1 .

For horospheres centered on white sides of boundary rectangles, we will also prove that the diameter is at most 1 . In order to do so, we will need to give specific instructions for expanding the cusps.

We would like to expand the cusps of $E(J)$ to a halfway point along each edge of the polyhedra. It turns out that even though an edge is infinitely long, there is a natural way to define its midpoint.

Definition 3.6. Let $T \subset \mathbb{H}^{3}$ be an ideal triangle. For each edge $e$ of $T$, define the midpoint to be the point $m \in e$ such that the geodesic from $m$ to the opposite vertex is perpendicular to $e$. (This point is unique, for otherwise we would have a triangle with one ideal vertex and two right angles.)

Now, each edge $e$ in the polyhedral decomposition of $E(J)$ borders on two shaded faces, $S_{1}$ and $S_{2}$, with each $S_{i} \subset P_{i}$. (See Figure 4.) It is easy to check that the two definitions of the midpoint of $e$, coming from $S_{1}$ and $S_{2}$, coincide. This is because $P_{1}$ and $P_{2}$ are symmetric by a reflection in the white faces of the projection diagram. The reflection preserves angles, so it also preserves the midpoint of $e$. Thus we have a well-defined midpoint of each edge of $E(J)$.

When two ideal triangles $T_{1}$ and $T_{2}$ are symmetric across an edge $e$, we have an alternate way of seeing the midpoint $m$ in $e$. Namely, the two ideal triangles glue up to form an ideal quadrilateral. One diagonal is $e$, and the other diagonal $d$ intersects $e$ 
at $m$. (See Figure 8.) By this approach, we see that when $T_{1}$ is in standard position and $e$ is vertical, $m$ is at Euclidean height 1 .

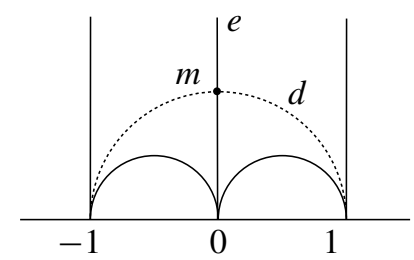

Figure 8 . When a triangle is in standard position, the midpoint of a vertical edge lies at height 1.

We are now ready to expand all the cusps of $E(J)$. Pick an order for the cusps, $K_{1}, K_{2}, \ldots, K_{r}$, and expand one $K_{i}$ at a time, starting with $K_{1}$. Continue expanding the horoball neighborhood of $K_{i}$ until it either meets another horoball, or meets the midpoint of some edge into $K_{i}$.

Lemma 3.7. Arrange $P_{1}$ or $P_{2}$ in $\mathbb{H}^{3}$ so that some shaded face is in standard position, and let $H$ be a horoball centered on a white side of a boundary rectangle of $P_{i}$. If the interior of $H$ does not contain the midpoint of any edge of $E(J)$, the diameter of $H$ can be at most 1 .

Proof. The center of $H$ on $S_{\infty}^{2}$ is an ideal vertex of some translate of $P_{i}$, and so at this ideal vertex, two white faces and two shaded faces meet and intersect $H$ in a rectangle. Recall that these faces are all totally geodesic, by Theorem 2.4. Since $H$ is centered on a white side of a boundary rectangle, we know one of these white faces is actually vertical. That is, one white face $V$ meeting the center of $H$ lies in a vertical plane in $\mathbb{H}^{3}$, bordered by a line $\ell \subset \mathbb{C}$. The other white face, $W$, must also lie in a geodesic plane $P$ in $\mathbb{H}^{3}$. $P$ cannot also be vertical (since $H$ is not centered at $\infty$ ), so it looks like a Euclidean half-sphere, tangent to the vertical plane containing $V$. Thus the boundary of $P$ at infinity is a circle $C$. (See Figure 9.)

Consider the white face $W$. Since white faces of $P_{i}$ only meet white faces at ideal vertices, other white faces meeting $W$ will lie on geodesic planes in $\mathbb{H}^{3}$ tangent to $P$. Thus they extend to give circles tangent to the circle $C$. In particular, these circles have disjoint interiors, and so the interior of $C$ must be disjoint from the two vertical planes containing the white sides of the boundary rectangle about the vertex of $P_{i}$ at $\infty$. But because we put a shaded side in standard position, these vertical planes are of Euclidean distance 1 apart. Thus the diameter of $C$ can be no more than 1 .

Now consider the two shaded faces meeting at the center of the horosphere $H$. At most one of these shaded faces can lie in a vertical plane. Let $S$ be one of these shaded faces that does not lie in a vertical plane. Let $e$ be the edge between $S$ and 


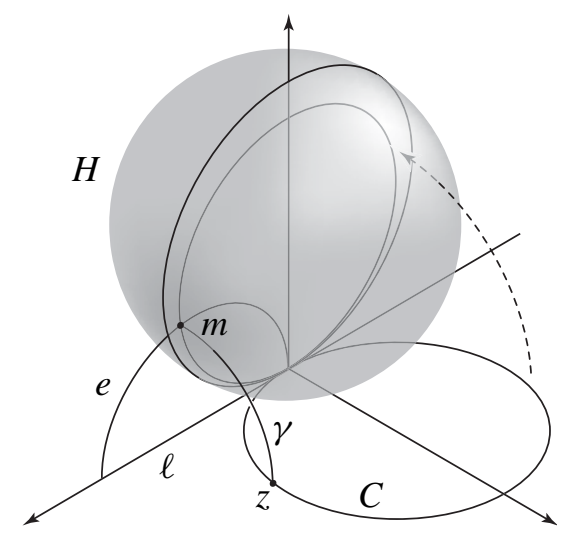

Figure 9. If the diameter of $H$ is greater than 1 , a $90^{\circ}$ rotation about the side of the boundary rectangle shows that the midpoint of $e$ lies inside $H$.

the vertical white face $V$, and let $z$ be the vertex of $S$ opposite $e$. Note that $z$ is an endpoint of the edge given by the intersection of $S$ with the white face $W$. (The other endpoint is the center of $H$.) Thus $z$ lies on the circle $C$.

Consider the midpoint $m$ of $e$. By Definition 3.6, the geodesic $\gamma$ from $z$ to $m$ meets $e$ at a right angle. But $\gamma$ must lie in $S$, which by Theorem 2.4 meets the face $V$ at a right angle, and hence $\gamma$ is normal to the entire vertical plane containing $V$. Thus $\gamma$ is a Euclidean quarter circle, centered on the line $\ell$ in $\mathbb{C}$ that lies under the vertical white face $V$. Then note that a $90^{\circ}$ rotation about $\ell$ takes the point $z$ to $m$. But this same $90^{\circ}$ rotation about $\ell$ will take the circle $C$ to the vertical plane over $\ell$. If the diameter of $H$ is greater than 1, the circle $C$, of diameter at most 1, will be contained inside $H$. Since $z$ is on $C$, in this case the rotated point $m$ will be contained inside $H$. (See Figure 9.) Hence if we do not allow $H$ to contain $m$, the diameter of $H$ can be at most 1 .

Theorem 3.8. Expand all the cusps of $E(J)$ as above. Then the midpoint of every edge of $E(J)$ will lie at the point of tangency of two horospheres.

Proof. First, we would like to show that an expanding horoball about a given cusp of $E(J)$ will simultaneously meet the midpoints of all the edges into that cusp. To that end, consider the horoball $H_{\infty}$ about $\infty$, normalized so that a shaded face $S$ is in standard position. $S$ forms a side of a boundary rectangle of Euclidean width 1 . The opposite side of this rectangle must be another shaded side of Euclidean width 1 . Continuing in the $\boldsymbol{w}$ direction, we see there is an infinite strip consisting of boundary rectangles lined end to end, and each shaded side in this strip has Euclidean width 1. 
We can now reflect this infinite strip by an involution in the white faces, obtaining an infinite strip whose sides are separated by $2 s$. By Lemma 2.6, translation by $2 s$ is an element of the covering transformation group for any cusp of $E(J)$. Thus every shaded face intersecting $H_{\infty}$ has Euclidean width 1 . Consequently, the midpoint of every vertical edge is at height $h=1$. (See Figure 8.)

As we expand the horoball $H_{\infty}$ about $\infty$, the expansion stops before height 1 only if $H_{\infty}$ becomes tangent to another horoball $H$ of diameter greater than 1 . But by Lemmas 3.5 and 3.7, all horoballs obtained with our expansion instructions have diameter at most 1 . Thus $H_{\infty}$ can be expanded until it reaches height $h=1$ and meets the midpoint of every vertical edge. By symmetry, the horosphere centered at the other endpoint of a vertical edge also meets the midpoint of that edge.

Corollary 3.9. Expand the cusps of $E(J)$ by the above procedure. Then in any boundary rectangle, $\ell(\boldsymbol{s})=1$ and $\ell(\boldsymbol{w}) \geq 1$.

Proof. Consider a boundary rectangle on a maximal cusp of $E(J)$, positioned so that a shaded face $S$ adjacent to this rectangle is in standard position. By Theorem 3.8, the horosphere $H_{\infty}$ about $\infty$ meets the midpoints of the vertical edges of $S$. In standard position, these midpoints lie at $h=1$, where Euclidean lengths correspond to hyperbolic lengths. Thus $\ell(s)$ is the Euclidean width of $S$, namely 1 .

Theorem 3.8 also implies that above every corner of a boundary rectangle, horosphere $H_{\infty}$ is tangent to a horosphere of diameter 1 . Since these horospheres are disjoint, we can conclude that $\ell(\boldsymbol{w}) \geq 1$.

3.3. Surgery consequences. By combining Corollary 3.9 with Theorem 2.7 , we can compute explicit lower bounds for the lengths of surgery curves.

Theorem 3.10. For each cusp $K_{i}$ of $E(J)$, pick a surgery slope $s_{i}$ that represents a non-trivial filling of the original link $K$. If $K_{i}$ is a knot strand cusp, let $n_{i}$ be the number of twist regions visited by the corresponding strand of $K$; if $K_{i}$ is a crossing circle cusp, let $n_{i}$ be the number of crossings in the corresponding twist region. Then

(1) on a knot strand cusp, $\ell_{g}\left(s_{i}\right) \geq n_{i}$, and

(2) on a crossing circle cusp, $\ell_{g}\left(s_{i}\right) \geq \sqrt{n_{i}^{2}+1}$.

Proof. For part (1), Theorem 2.7 implies that any non-trivial surgery curve on $K_{i}$ is of the form $p_{i} n_{i} \boldsymbol{w}+q_{i} \boldsymbol{s}$ for integers $p_{i} \neq 0$ and $q_{i}$. Then since $\ell(\boldsymbol{s})=1$ and $\ell(\boldsymbol{w}) \geq 1$, and $\boldsymbol{s}$ and $\boldsymbol{w}$ are perpendicular, any such curve will have length at least $\sqrt{p_{i}^{2} n_{i}^{2}+q_{i}^{2}}$. This is minimal when $q_{i}=0$ and $p_{i}= \pm 1$. In this case, $\ell\left(s_{i}\right) \geq n_{i}$.

For part (2), Theorem 2.7 implies the surgery curve is $w \pm n_{i} s$, which has length at least $\sqrt{n_{i}^{2}+1}$. 
We are now ready to give our geometric proof of Theorem 1.7, which we restate.

Theorem 1.7. Let $K$ be a link in $S^{3}$ with a prime, twist-reduced diagram $D(K)$. Suppose that every twist region of $D(K)$ contains at least 6 crossings and each component of $K$ passes through at least 7 twist regions (counted with multiplicity). Then every non-trivial Dehn filling of all the components of $K$ is hyperbolike.

Proof. Since each knot strand cusp crosses at least 7 twist regions, Theorem 3.10 says that the surgery curve on that cusp has length at least 7. Since each twist region contains at least 6 crossings, the surgery curve on the corresponding crossing circle has length at least $\sqrt{6^{2}+1}>6$. Thus the surgery curve on every component of $\partial E(J)$ has length greater than 6 . Therefore, by the 6-Theorem, the surgered manifold is hyperbolike.

Our information about the cusp shapes of $E(J)$ also allows for the following extension of Theorem 1.7.

Theorem 3.11. Let $K$ be a link in $S^{3}$ with a prime, twist-reduced diagram $D(K)$. Suppose that every twist region of $D(K)$ contains at least 7 crossings and each component of $K$ passes through at least 7 twist regions (counted with multiplicity). Then every non-trivial Dehn filling of all the components of $K$ yields a manifold that admits a negatively curved Riemannian metric.

Proof. Under these hypotheses, every surgery curve on $\partial E(J)$ will have length at least 7 . In particular, because every surgery curve is strictly longer than $2 \pi$, Gromov and Thurston's $2 \pi$-Theorem [4] implies that the Dehn filled manifold admits a metric of negative sectional curvatures.

The Geometrization Conjecture implies that the conclusions of Theorems 1.7 and 3.11 are in fact equivalent. One advantage of Theorem 3.11, despite its stronger hypotheses, is that the negatively curved metric can be explicitly constructed. In fact, by estimating the sectional curvatures and volume of this negatively curved metric, one may obtain explicit estimates on the hyperbolic volume of $K$ and its Dehn fillings [8].

\section{Angled polyhedra and normal surfaces}

The next two sections give combinatorial proofs of Theorems 1.4, 1.5, and 1.7, by using a combinatorial notion of length. In this proof, we make use of a number of results from the theory of normal and admissible surfaces in angled polyhedra, much of it developed by Marc Lackenby [12, Section 4]. Lackenby worked with the dual structure of angled spines. We find it more convenient to work with polyhedra, so in this section we will translate his definitions and theorems into polyhedral language. 


\subsection{Normal and admissible surfaces}

Definition 4.1. For the purposes of this paper, a polyhedron is a 3-ball $P$ with a specified connected graph $\Gamma$ embedded in $\partial P$, such that

(1) each vertex of $\Gamma$ has valence at least 3 ,

(2) each edge of $\Gamma$ has ends on distinct vertices, and

(3) each region of $\partial P \backslash \Gamma$ is bounded by at least 3 edges.

$P$ inherits vertices and edges from $\Gamma$, and the faces of $P$ are regions of $\partial P \backslash \Gamma$. An ideal polyhedron is a polyhedron with the vertices removed.

Remark. This definition of an ideal polyhedron is actually slightly stronger than Lackenby's dual definition of a thickened spine, in that condition (3) prohibits our polyhedra from having bigon faces. This stronger definition is sufficient for our purposes (certainly, the ideal polyhedra $P_{1}$ and $P_{2}$ constructed in Section 2 have no bigon faces), and allows for stronger statements of some results.

Let $M$ be a manifold subdivided into ideal polyhedra. To see how the ideal vertices fit together to tile $\partial M$, we truncate all the ideal vertices. This gives new polyhedra, with two kinds of faces: interior faces that are truncated copies of the original faces, and boundary faces that come from the truncated vertices. We also obtain two kinds of edges: interior edges that come from the original truncated edges, and boundary edges along the boundary faces.

In order to define a combinatorial length for a curve on $\partial M$, we actually need to consider surfaces inside the manifold with that curve as boundary. Thus we review some results from the theory of normal and admissible surfaces.

Let $(F, \partial F) \subset(M, \partial M)$ be an embedded essential surface (a sphere not bounding a ball, or an incompressible, boundary-incompressible surface). The theory of normal surfaces, originally developed by Haken [10] and generalized and expanded in many directions, says that $F$ can be isotoped until its intersections with the polyhedra (or handles) have a particularly nice form. Specifically, we can get $F$ to intersect each polyhedron in a collection of disjoint, embedded disks, with each disk positioned so that its boundary curve $\gamma$ has several nice properties:

Definition 4.2. Let $P$ be a truncated ideal polyhedron. A simple closed curve $\gamma \subset \partial P$ is called normal if

(1) $\gamma$ is transverse to the edges of $P$,

(2) no arc of $\gamma$ in a face of $P$ has endpoints on the same edge, or on an interior edge and an adjacent boundary edge,

(3) $\gamma$ does not lie entirely in a face of $P$, 
(4) $\gamma$ intersects each edge at most once, and

(5) $\gamma$ intersects each boundary face at most once.

The disk in $P$ bounded by a normal curve $\gamma$ is called a normal disk. See Figure 10 (a) for several examples.
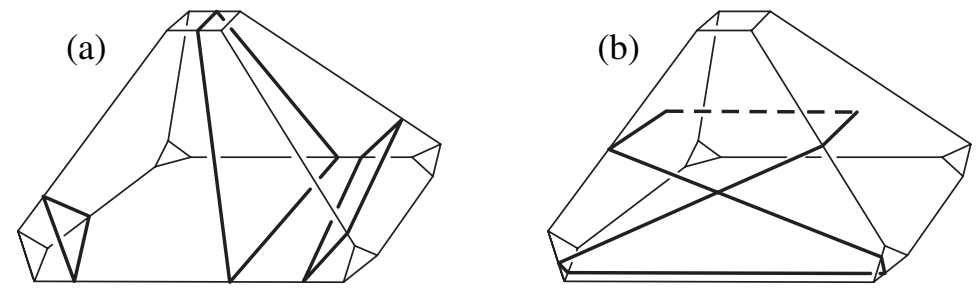

Figure 10. (a) Normal disks in a truncated polyhedron. (b) An admissible disk.

Notation. To avoid confusion with longer arcs on $\partial M$, we will refer to the arcs of intersection between a normal curve $\gamma$ and the faces of $P$ as segments. (Thus an arc can consist of many segments.) Segments of $\gamma$ lying in interior faces of $P$ will be called interior segments, and the segments lying in boundary faces will be called boundary segments.

In order to prove word-hyperbolicity, we actually need to work with a more general class of surfaces that cannot be normalized. These surfaces may not be embedded, and may even have boundary components in the interior of $M$.

Definition 4.3. Let $P$ be a truncated ideal polyhedron. An immersed disk $D \subset P$ is called admissible if

(1) $\partial D \backslash \partial P$ is a (possibly empty) collection of embedded arcs with endpoints inside interior faces of $P$,

(2) $\partial D \cap \partial P$ is an immersed closed curve or an immersed collection of arcs,

(3) if $\partial D \cap \partial P$ is a closed curve, it satisfies conditions (1)-(3) of Definition 4.2 of a normal curve,

(4) each arc component of $\partial D \cap \partial P$ satisfies conditions (1)-(2) of Definition 4.2, and

(5) each segment of $\partial D$ in a face of $P$ is embedded.

An example is shown in Figure 10(b). We call an immersed surface $F \subset M$ an admissible surface if it intersects each polyhedron in a collection of admissible disks. 
4.2. Angle structures and combinatorial area. The theory of normal surfaces becomes much more powerful if one has information about the dihedral angles of ideal polyhedra.

Definition 4.4. Let $M$ be a manifold with boundary. An angled polyhedral decomposition of $M$ is a subdivision of $M \backslash \partial M$ into ideal polyhedra, glued along their interior faces. Each interior edge of each angled polyhedron is assigned an internal angle $\alpha_{i} \in(0, \pi)$ and an external angle $\epsilon_{i}=\pi-\alpha_{i}$, such that

(1) around each edge of $M, \sum \alpha_{i}=2 \pi$, and

(2) in each polyhedron, for a normal curve $\gamma$ that intersects only interior edges, $\sum_{\gamma} \epsilon_{i} \geq 2 \pi$, with equality if and only if $\gamma$ encircles a vertex.

Angle structures on a polyhedral decomposition of $M$ allow us to define the combinatorial area of a surface.

Definition 4.5. Let $D \subset P$ be an admissible disk in an angled polyhedron, with the boundary faces of $P$ lying on $\partial M$. Let $E_{1}, \ldots, E_{n}$ be the interior edges crossed by $\partial D$ (counted with multiplicity), and let $\epsilon_{1}, \ldots, \epsilon_{n}$ be the corresponding external angles. Then define the combinatorial area of $D$ to be

$$
a(D)=\sum_{i=1}^{n} \epsilon_{i}+\pi|\partial D \cap \partial M|-2 \pi+3 \pi|\partial D \backslash \partial P| .
$$

For an admissible surface $F \subset M, a(F)$ is defined by summing the areas of its admissible disks.

For disks with $\partial D \subset \partial P$, this definition matches the formula for hyperbolic area. For a polygon $T \subset \mathbb{H}^{2}$ with external angles $\epsilon_{i}, a(T)=\sum \epsilon_{i}-2 \pi$. (See, for example, Corollary 2.4.15 of [25].) Ideal vertices have internal angle 0 and thus add $\pi$ to the area, just as each component of $\partial D \cap \partial M$ adds $\pi$ to combinatorial area. As for the coefficient $3 \pi$ per component of $\partial D \backslash \partial P$, it was chosen by Lackenby to make the combinatorial area of $D$ automatically positive whenever $\partial D$ passes through the interior of $P$.

In fact, there are only two types of admissible disks whose area is 0 ; both of them happen to be normal. The first is a vertex link cutting off a boundary face; its area is 0 by Definition 4.4. The second is a boundary bigon cutting off an interior edge; it has area 0 because its boundary curve only picks up area from two boundary faces. They are shown in Figure 11.

Lemma 4.6. Let $D \subset P$ be an admissible disk in an angled polyhedron. If $D$ is not a vertex link or a boundary bigon, then $a(D)>0$. 

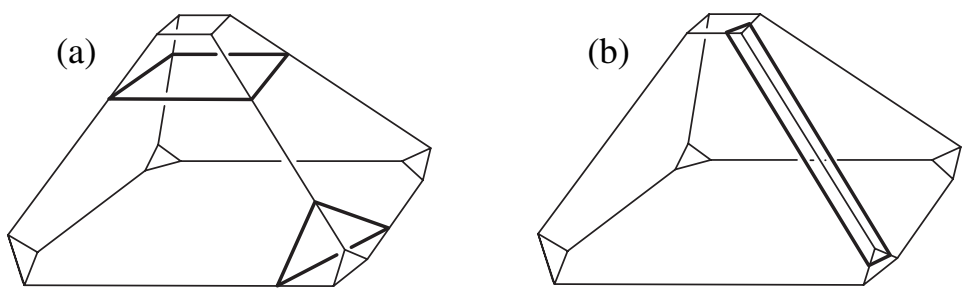

Figure 11. (a) Vertex links. (b) A boundary bigon.

Proof. If $D$ is normal, Lackenby [13, Lemma 4] proves that $a(D)>0$ unless $D$ is a vertex link or a bigon. If $D$ is not normal, Lackenby [12, Lemma 4.2] proves that $a(D)>0$. In both cases, the proofs rely on condition (2) of Definition 4.4 of an angled polyhedron and the observation that if $\partial D$ self-intersects or crosses an edge multiple times, the area can actually be reduced by surgering the disk.

Remark. Lemma 4.6 is one place where our definition of a polyhedron, which is stronger than Lackenby's definition of an angled spine because it rules out bigon faces, becomes convenient. Bigon faces of the polyhedra allow normal disks other than boundary bigons or vertex links to have zero area [12, Lemma 4.2]; in our scenario, every other admissible disk has strictly positive area.

The analogy between hyperbolic area and combinatorial area extends to the following combinatorial version of the Gauss-Bonnet theorem [12, Proposition 4.3].

Proposition 4.7 (Gauss-Bonnet Theorem). Let $F \subset M$ be an admissible surface in a manifold with an angled polyhedral decomposition. Let Length $(\partial F \backslash \partial M)$ be the number of arcs of intersection between $\partial F \backslash \partial M$ and the polyhedra. Then

$$
a(F)=-2 \pi \chi(F)+2 \pi \operatorname{Length}(\partial F \backslash \partial M) .
$$

Combinatorial area in angled polyhedra has powerful consequences. Among them is the following stronger version of a result of Lackenby [12, Corollary 4.6]:

Theorem 4.8. Let $M$ be an orientable 3-manifold with an angled polyhedral decomposition. Then $\partial \mathrm{M}$ is composed of tori, and $M$ is hyperbolic.

Proof. To prove the first assertion, observe that each component of $\partial M$ is tiled by boundary faces of the polyhedra. Just inside each boundary face, a polyhedron has a normal disk of area 0 . These vertex links glue up to form a closed, boundary-parallel normal surface $F$ of area 0 . By Proposition $4.7, \chi(F)=0$, and since $M$ is orientable, $F$ must be a torus. 
By Thurston's Hyperbolization Theorem [24], a manifold with boundary consisting of tori is hyperbolic if and only if it contains no essential spheres, disks, tori, or annuli. If M contains such a surface $S$, then it contains one in normal form. If $S$ is a sphere or disk, then it has positive Euler characteristic, hence negative area. Thus spheres and disks cannot occur.

A normal torus $T \subset M$ has area 0 and thus, by Lemma 4.6, must be composed of normal disks of area 0 . Since $T$ has no boundary, these must all be vertex links, which glue up to form a boundary-parallel torus. Similarly, a normal annulus $A \subset M$ must be composed entirely of bigons, since a bigon cannot be glued to a vertex link. But a chain of bigons forms a tube around an edge of $M$, which is certainly not essential. Thus we can conclude that $M$ is hyperbolic.

4.3. Combinatorial length and surgery results. Lackenby's crucial insight [12] is that one can use the combinatorial area of surfaces in a manifold $M$ to define a combinatorial length of curves on $\partial M$, and that this notion of length turns out to be closely related to geometric length on a maximal cusp.

Definition 4.9. Let $P$ be an angled polyhedron, and let $D \subset P$ be an admissible disk that intersects at least one boundary face. Let $\gamma$ be a segment of $\partial D$ in a boundary face of $P$. Then we define the length of $\gamma$ relative to $D$ to be

$$
\ell(\gamma, D)=\frac{a(D)}{|\partial D \cap \partial M|} .
$$

Definition 4.10. For a manifold $M$ with an angled polyhedral decomposition, let $\gamma$ be a (possibly non-closed) immersed arc in $\partial M$. We call $\gamma$ a simplicial arc if

(1) $\gamma$ is disjoint from the vertices of $\partial M$,

(2) the endpoints of $\gamma$ (if any) lie on edges of $\partial M$,

(3) each segment of $\gamma$ in a boundary face is embedded, and

(4) no segment of $\gamma$ in a boundary face has endpoints on the same edge.

We can now define the combinatorial length of simplicial arcs on $\partial M$ by considering all the possible inward extensions of the arc.

Definition 4.11. Let $\gamma \subset \partial M$ be a simplicial arc. Let $\gamma_{1}, \ldots, \gamma_{n}$ be the boundary segments that make up $\gamma$, ordered along a parametrization of $\gamma$. For each $i$, let $D_{i}$ be an admissible disk in the corresponding polyhedron, whose boundary contains $\gamma_{i}$. Then $H=\bigcup_{i=1}^{n} D_{i}$ is called an inward extension of $\gamma$ if

(1) $\partial D_{i}$ agrees with $\partial D_{i+1}$ on the shared face of their polyhedra, and

(2) if $\gamma$ is closed, $\partial D_{n}$ agrees with $\partial D_{1}$ on the common face. 
We define the combinatorial length of $\gamma$ to be

$$
\ell_{c}(\gamma)=\inf \left\{\sum_{i=1}^{n} \ell\left(\gamma_{i}, D_{i}\right)\right\},
$$

where the infimum is taken over all inward extensions of $\gamma$. The subscript in $\ell_{c}(\gamma)$ serves to distinguish combinatorial length from the geometric cusp length $\ell_{g}(\gamma)$ used in Section 3. When the meaning is clear, we will simply use $\ell(\gamma)$.

Definition 4.12. Let $s$ be a slope on a boundary component of $M$. Then define

$$
\ell_{c}(s)=\inf \left\{\ell_{c}(\gamma)\right\}
$$

the infimum being taken over all closed simplicial curves $\gamma \subset \partial M$ that represent non-zero multiples of slope $s$.

The point of this string of definitions is to imply the following lemma, which is essentially Proposition 4.8 of [12], rewritten in terms of polyhedra instead of spines.

Lemma 4.13. Let $M$ be a manifold with an angled polyhedral decomposition, and let $F \subset M$ be an admissible surface. Let $C_{1}, \ldots, C_{m}$ be the components of $\partial F \cap \partial M$, each $C_{j}$ representing a non-zero multiple of some slope $s_{i(j)}$. Then

$$
a(F) \geq \sum_{j=1}^{m} \ell_{c}\left(s_{i(j)}\right) .
$$

Proof. The admissible disks of $F$ bordering on each $C_{j}$ form one inward extension of $C_{j}$. Definition 4.9 has us divide the area of each disk by the number of its intersections with $\partial M$, so we do not end up double-counting any area.

As a consequence of Lemma 4.13, surfaces with long boundary have large combinatorial area, hence large genus. This yields the following combinatorial analogue of the 6-Theorem, stated as Theorem 4.9 of [12].

Theorem 4.14 (Lackenby). Let $M$ be a manifold with an angled polyhedral decomposition. Let $s_{1}, \ldots, s_{n}$ be a collection of slopes on $\partial M$, with one $s_{i}$ on each component of $\partial M$. If $\ell_{c}\left(s_{i}\right)>2 \pi$ for each $i$, then the manifold obtained by Dehn filling $M$ along the slopes $s_{1}, \ldots, s_{n}$ is hyperbolike.

In fact, Lackenby's machinery allows for an extension of his theorem to surgeries along only some components of $\partial M$. 
Theorem 4.15. Let $M$ be a manifold with an angled polyhedral decomposition. Let $s_{1}, \ldots, s_{m}$ be a collection of slopes on some, but not all, of the boundary tori. If $\ell_{c}\left(s_{i}\right)>2 \pi$ for each $i$, then the manifold obtained by Dehn filling $M$ along the slopes $s_{1}, \ldots, s_{m}$ is hyperbolic.

Proof. By Thurston's Hyperbolization Theorem [24], proving that the Dehn filled manifold is hyperbolic amounts to ruling out essential spheres, disks, tori, and annuli. Any such surface $F$ must intersect at least one of the solid tori added during the surgery process, because $M$ is hyperbolic by Theorem 4.8. Thus $F$ contains a punctured surface $G \subset M$, whose punctures (not counting the original boundary components of $F$ ) represent surgery slopes $s_{i(1)}, \ldots, s_{i(k)}$ of length greater than $2 \pi$. We can place $G$ in normal form in the angled polyhedra and compute its combinatorial area. Then

$$
\begin{aligned}
a(G) & =-2 \pi \chi(G) & & \text { by Proposition } 4.7 \\
& \leq 2 \pi|\partial G \backslash \partial F| & & \text { given the choices of } F \\
& <\sum_{j=1}^{|\partial G \backslash \partial F|} \ell\left(s_{i(j)}\right) & & \text { by assumption } \\
& \leq a(G) & & \text { by Lemma 4.13, }
\end{aligned}
$$

obtaining a contradiction.

Juxtaposing Theorem 3.2 with Theorems 4.14 and 4.15, one can see that for the purpose of ruling out exceptional surgeries, $\ell_{c}\left(s_{i}\right)$ corresponds to $\frac{\pi}{3} \ell_{g}\left(s_{i}\right)$. It turns out that on the cusps of $E(J)$, geometric and combinatorial length have a similar correspondence (compare Theorem 3.10 with Corollary 5.12). This yields a second, combinatorial, proof of Theorem 1.7.

\section{Normal surfaces in the augmented link polyhedra}

In this section, we apply the normal surface theory of Section 4 to the ideal polyhedral decomposition of the augmented link complement $E(J)$, constructed in Section 2. Recall that by Theorem 2.4, $P_{1}$ and $P_{2}$ are convex ideal polyhedra in $\mathbb{H}^{3}$, so they satisfy the definition of an angled polyhedron. (See [21, Theorem 1].) In fact, they are examples of a special type of ideal polyhedron, which we call rectangular-cusped. If we truncate the ideal vertices, as we did with $P_{1}$ and $P_{2}$ in Section 2, the resulting boundary faces subdivide $\partial M$ into rectangles. 


\subsection{Rectangular-cusped polyhedra}

Definition 5.1. Let $P$ be an angled ideal polyhedron (see Definition 4.4) in which we have truncated the ideal vertices. We say that $P$ is rectangular-cusped if

(1) each boundary face of $P$ (each face of $P \cap \partial M$ ) meets 4 interior edges, and

(2) each interior edge is labeled with angle $\pi / 2$.

Rectangular-cusped polyhedra have two convenient features. First, their interior faces can be two-colored, in a similar fashion to the white and shaded faces of $P_{1}$ and $P_{2}$. Around each rectangular boundary face, opposite interior faces have the same color. Second, making all dihedral angles equal to $\pi / 2$ ensures that all combinatorial areas are multiples of $\pi / 2$.

In addition to the vertex links and boundary bigons of area 0 (see Figure 11), we need to define a third kind of special admissible disk.

Definition 5.2. Let $P$ be a truncated ideal polyhedron. An admissible disk $D \subset P$ is called an ideal triangle if

(1) $\partial D \subset \partial P$,

(2) $\partial D$ intersects the boundary faces of $P$ exactly three times, and

(3) $\partial D$ is disjoint from the interior edges of $P$.

Two examples are shown in Figure 12. Note that an ideal triangle $D$ has area $a(D)=\pi$ and length $\ell(\gamma, D)=\pi / 3$ for each segment $\gamma$ of $\partial D \cap \partial M$.

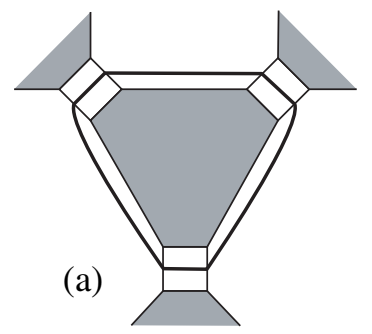

(b)

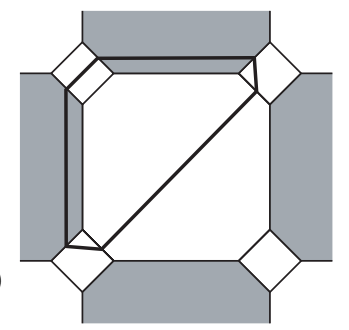

Figure 12. Two ideal triangles in a rectangular-cusped polyhedron.

Proposition 5.3. Let $D \subset P$ be an admissible disk in a rectangular-cusped polyhedron, such that $\partial D$ passes through at least one boundary face. Let $\gamma \subset \partial M$ be a boundary segment of $\partial D$. If $D$ is not a bigon or an ideal triangle,

$$
\ell(\gamma, D) \geq \frac{\pi}{2} .
$$


Proof. We consider different cases, conditioned on $n=|\partial D \cap \partial M|$. By Definition $4.9, \ell(\gamma, D)=a(D) / n$.

Case 1: $n=1$. For this case, we need to prove that $a(D) \geq \pi / 2$. An admissible disk with one component of $\partial D \cap \partial M$ cannot be a vertex link or boundary bigon, so by Lemma 4.6, $a(D)>0$. Since all areas in a rectangular-cusped polyhedron are multiples of $\pi / 2, a(D) \geq \pi / 2$.

Case 2: $n=2$. For this case, we need to prove that $a(D) \geq \pi$. If $a(D)=0, D$ is a boundary bigon, excluded by the hypotheses. So we need to rule out the possibility that $a(D)=\pi / 2$.

If such a disk were to occur, it would have to have $\partial D \subset \partial P$, and $\partial D$ would have to intersect exactly one interior edge. Then $\partial D$ passes through three interior faces, which cannot all have the same color because two of them share an edge. Thus a segment $\gamma_{1} \subset \partial D$ in a boundary face must connect adjacent interior faces, for otherwise all three interior faces would have the same color. See Figure 13 for a schematic picture.

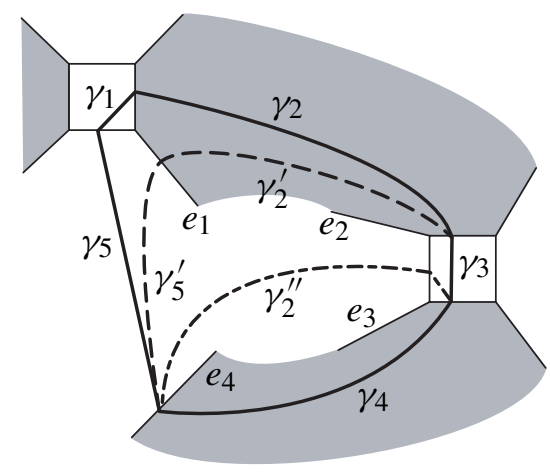

Figure 13. Schematic picture for Case 2 of Proposition 5.3.

We can pull $\gamma_{1}$ off the boundary face and have it intersect interior edge $e_{1}$. This creates a new disk $D^{\prime}$ with one segment on $\partial M$ and area 0 , since this isotopy reduced the area by $\pi / 2$. If $D^{\prime}$ were admissible, it would be a counterexample to Case 1 . Thus $\partial D^{\prime}$ must violate some condition of admissibility. The only way this can happen is if one of the new segments of $\partial D^{\prime}, \gamma_{2}^{\prime}$ or $\gamma_{5}^{\prime}$, has both endpoints on the same edge, or on adjacent interior and boundary edges. But since $D$ is admissible, $e_{1}$ and $e_{4}$ must be distinct edges, so $\gamma_{5}^{\prime}$ has endpoints on distinct edges.

Thus $\gamma_{2}^{\prime}$ connects adjacent interior and boundary edges, and so $e_{1}=e_{2}$. We can then isotope $\gamma_{2}^{\prime}$ across this interior edge, creating a new disk $D^{\prime \prime}$ that has just one intersection with $\partial M$ and one intersection with an interior edge. Since $\gamma_{4}$ and the new segment $\gamma_{2}^{\prime \prime}$ lie in adjacent faces of $P$, we have $e_{3}=e_{4}$. Then $\gamma_{4}$ connects adjacent 
interior and boundary edges, contradicting the assumption that $D$ was admissible. Therefore, such a disk $D$ does not exist.

Case 3: $n=3$. For this case, we need to prove that $a(D) \geq 3 \pi / 2$. The three components of $\partial D \cap \partial M$ already ensure that $a(D) \geq \pi$. So if $\partial D$ also intersects an interior edge or the interior of $P$, we have $a(D) \geq 3 \pi / 2$. Otherwise, $D$ is an ideal triangle, excluded by the hypotheses.

Case 4: $n \geq 4$. For this case,

$$
a(D) \geq n \cdot \pi-2 \pi \geq \frac{n}{2} \cdot \pi
$$

proving the lemma.

Thus ideal triangles are the only admissible disks of nonzero area that contribute less than $\pi / 2$ to combinatorial length. To obtain the best possible bounds on the length of surgery curves, we need to find out more about how these triangles fit into polyhedra $P_{1}$ and $P_{2}$ that decompose the link complement $E(J)$.

\subsection{More on ideal triangles}

Lemma 5.4. Let $P$ be a truncated angled ideal polyhedron, and let $D \subset P$ be an ideal triangle. Then all the segments of $\partial D$ lie in distinct faces of $\partial P$, and $D$ is normal.

Proof. $\partial D$ consists of six segments, alternating between boundary and interior faces. Label them $\gamma_{1}, \ldots, \gamma_{6}$. Suppose that two of these segments (say, $\gamma_{1}$ and $\gamma_{3}$ ) lie in the same face $F$. Then the endpoints of $\gamma_{2}$, which must lie in different edges because $D$ is admissible, both meet $F$. But if we connect the two endpoints of $\gamma_{2}$ to each other through $F$, we will obtain a normal disk with negative combinatorial area, contradicting Lemma 4.6.

Thus each $\gamma_{i}$ must lie in a different face, so $\partial D$ is embedded. Since $\partial D$ intersects each boundary face at most once and is disjoint from the interior edges altogether, $D$ must be normal.

For the rest of this section, we will work directly with the polyhedra $P_{1}$ and $P_{2}$, and the only manifolds we will consider are $E(J)$ and its Dehn fillings.

Definition 5.5. In polyhedra $P_{1}$ and $P_{2}$, we will classify ideal triangles into three types. A triangle of type $S$ is one that is parallel to a shaded face, as in Figure 12 (a). A triangle of type $W$ is one that is parallel to a white face, as in Figure 12(b). An ideal triangle parallel to no face of its polyhedron will be of type $N$.

Lemma 5.6. Let $D$ be an ideal triangle in $P \in\left\{P_{1}, P_{2}\right\}$. Let $\gamma_{1}, \ldots, \gamma_{6}$ be the segments of $\partial D$. Then the following hold: 
(1) If $D$ is of type $S$ or type $W$, then at least two of the $\gamma_{i}$ are parallel to interior edges of $P$.

(2) If $D$ is of type $N$, then no $\gamma_{i}$ is parallel to an interior edge, and the three interior faces of $P$ intersecting $\partial D$ are all white faces.

Proof. We consider two cases:

Case 1: $\partial D$ intersects both white and shaded faces. Then $D$ can be schematically represented by the left side of Figure 14. Label the stumps of interior edges $e_{1}, \ldots, e_{4}$, as in the figure; some of these are likely to be part of the same edge. Now, we can pull segment $\gamma_{2}$ off the boundary face and have $\partial D$ intersect edge $e_{2}$ instead. This creates a disk $D^{\prime}$ of area $\pi / 2$, which could a priori be normal. However, by Case 2 of Proposition 5.3, there are no normal disks that have two intersections with $\partial M$ and area $\pi / 2$. Thus $D^{\prime}$ fails some part of Definition 4.2.
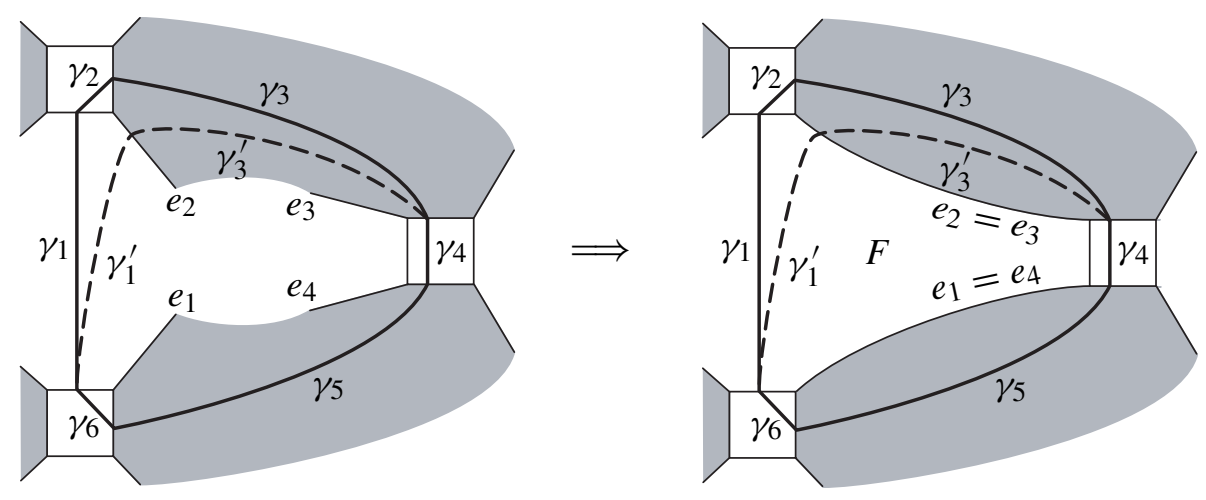

Figure 14. Schematic picture of an ideal triangle intersecting faces of both colors. The shading is generic, and might be reversed.

Since $D$ is normal by Lemma 5.4, the only way that $D^{\prime}$ can fail to be normal is if one of the new segments, $\gamma_{1}^{\prime}$ or $\gamma_{3}^{\prime}$, connects adjacent boundary and interior faces. If $\gamma_{1}^{\prime}$ violates normality, $e_{1}$ is the same edge as $e_{2}$. But then $\gamma_{3}$ and $\gamma_{5}$ must lie in the same face, contradicting Lemma 5.4.

If $\gamma_{3}^{\prime}$ violates normality by connecting adjacent boundary and interior faces, we can tighten $\partial D^{\prime}$ by removing its intersection with $e_{2}=e_{3}$. This creates a new disk $D^{\prime \prime}$ with area 0 . Segment $\gamma_{5}$ and the isotopic image of $\gamma_{3}$ lie in distinct faces because they are on opposite sides of edge $e_{4}$. So $D^{\prime \prime}$ is normal, and thus a boundary bigon. Then we can conclude that $e_{1}=e_{4}$, and the original disk $D$ was parallel to face $F$, into which we have pulled $\gamma_{3}$ (see Figure 14, right). So $D$ is of type S or W. Notice that both $\gamma_{3}$ and $\gamma_{5}$ are parallel to edges of $F$. 
Case 2: All interior faces intersecting $\partial D$ are the same color. If some segment $\gamma_{i}$ is parallel to an interior edge, we can isotope $\partial D$ across that edge, into a face of a different color, putting us in Case 1. Otherwise, if no $\gamma_{i}$ is parallel to an interior edge, the three interior faces must all be white. (Shaded faces are all triangles, in which any arc connecting distinct ideal vertices is parallel to an edge.) By Lemma 5.4, the segments $\gamma_{i}$ all lie in distinct faces, so since none of them is parallel to an edge, $D$ cannot be parallel to a face. Thus $D$ is of type $\mathrm{N}$, and satisfies conclusion (2) of the lemma.

Corollary 5.7. In an admissible surface in $E(J)$, an ideal triangle of type $N$ cannot be glued to a bigon or a triangle of type $S$.

Proof. Let $F$ be a shaded face of $P_{1}$ or $P_{2}$, and $D$ be a type $\mathrm{S}$ ideal triangle parallel to $F$. Since shaded faces are all triangles, every interior segment of $\partial D$ is parallel to an interior edge of $F$, hence an edge of $E(J)$. Similarly, both interior segments on the boundary of a bigon are parallel to an edge of $E(J)$. On the other hand, by Lemma 5.6 the boundary of a type $\mathrm{N}$ ideal triangle does not have any segments parallel to interior edges.

5.3. Progressive arcs and length estimates. We are now ready to estimate the combinatorial length of surgery slopes on $\partial E(J)$.

Definition 5.8. Let $T$ be a torus of $\partial E(J)$. Recall that, by Definition 2.5 , its universal cover $\widetilde{T}$ contains a lattice of shaded and white faces, generated by a basis $\langle\boldsymbol{s}, \boldsymbol{w}\rangle$. If $T$ is a crossing circle cusp, we will say that the $\boldsymbol{w}$ direction is meridional and the $s$ direction is longitudinal. If $T$ is a knot strand cusp, we will say that the $s$ direction is meridional and the $\boldsymbol{w}$ direction is longitudinal. (By Lemma 2.6, the meridian and longitude of $T$ are in fact aligned primarily in these directions.)

Thus if a segment $\gamma$ spans opposite edges of a boundary face $B \subset \partial E(J)$, it makes sense to talk of $\gamma$ lying in a meridional or longitudinal direction.

Definition 5.9. Let $P \in\left\{P_{1}, P_{2}\right\}$, and let $D \subset P$ be an admissible disk. Then $D$ can intersect a boundary face $B \subset \partial E(J)$ in one of three types of segments: a longitudinal segment, connecting opposite edges of $B$ in a longitudinal direction; a meridional segment, connecting opposite edges of $B$ in a meridional direction; or a diagonal segment, connecting adjacent edges of $B$.

To estimate the combinatorial length of surgery slopes on $\partial E(J)$ representing a surgery slope, it helps to divide a curve into smaller pieces. 
Definition 5.10. Let $T$ be a torus of $\partial E(J)$, and let $\gamma \subset T$ be a non-closed simplicial $\operatorname{arc}($ see Definition 4.10). Lift $\gamma$ to an arc $\widetilde{\gamma} \subset \widetilde{T}$, and cut $\widetilde{T}$ into vertical strips along meridional faces in the lattice. We say that $\gamma$ is a progressive arc if $\tilde{\gamma}$ is contained entirely in one of these vertical strips, and the endpoints of $\tilde{\gamma}$ lie on opposite sides of the strip.

In other words, a progressive arc on a crossing circle cusp has endpoints on consecutive white faces, and constitutes a step in the $s$ direction. A progressive arc on a knot cusp has endpoints on consecutive shaded faces, and constitutes a step in the $\boldsymbol{w}$ direction. In either case, a progressive arc $\gamma$ can consist of (a) a single longitudinal segment, (b) two diagonal segments connecting to different meridians, or (c) two diagonals with some number of meridional segments between them. These basic types are shown in Figure 15.
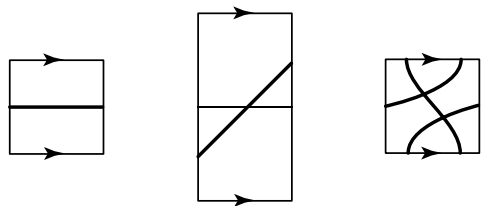

Figure 15. The three types of progressive arcs.

Lemma 5.11. Let $\gamma \subset \partial E(J)$ be a progressive arc. Then $\ell(\gamma) \geq \pi / 3$.

Proof. Let $H$ be an inward extension of $\gamma$ (see Definition 4.11). For each admissible disk $D_{i} \subset H$ bordering on a segment $\gamma_{i} \subset \gamma, \ell\left(\gamma_{i}, D_{i}\right)=0$ if and only if $D_{i}$ is a boundary bigon. By Proposition 5.3, every other type of disk contributes at least $\pi / 3$ to $\ell(\gamma)$. So the only way to have $\ell(\gamma)<\pi / 3$ is if $H$ consists only of bigons. However, a string of bigons circles around a single edge of $E(J)$, which means that its intersection with a component of $\partial E(J)$ cannot be a progressive arc.

Corollary 5.12. Let $T$ be a torus of $\partial E(J)$, and let s be a non-trivial surgery slope on $T$. If $T$ comes from a crossing circle $C_{i}$, let $n$ be the number of crossings in region $R_{i}$; if $T$ comes from a component $K_{j}$ of $K$, let $n$ be the number of twist regions visited by $K_{j}$, counted with multiplicity. Then, in either case,

$$
\ell_{c}(s) \geq \frac{n \pi}{3} .
$$

Proof. By Theorem 2.7, a surgery curve on a crossing circle corresponding to $n$ crossings must cross at least $n$ (white) meridional faces, and any surgery curve on a component of $K$ passing through $n$ twist regions with multiplicity must cross at least $n$ 
(shaded) meridional faces. Specifically, we can say that they must each contain at least $n$ progressive arcs. Thus the result follows from Lemma 5.11.

For surgery curves on a crossing circle cusp, which by Theorem 2.7 look like $n \boldsymbol{s} \pm \boldsymbol{w}$ in the basis $\langle\boldsymbol{s}, \boldsymbol{w}\rangle$, we can obtain a slightly better estimate.

Proposition 5.13. Let $s \subset \partial E(J)$ be a surgery slope on a crossing circle cusp that yields $n$ crossings. Then we have the strict inequality

$$
\ell_{c}(s)>\frac{n \pi}{3} \text {. }
$$

Proof. By Corollary 5.12, we must only rule out equality. Equality occurs when a simplicial curve $c$ representing $s$ contains exactly $n$ progressive arcs, an inward extension of $c$ picks up length exactly $\pi / 3$ per progressive arc, and any part of $c$ not covered by progressive arcs contributes zero length. Consider such a curve.

If a progressive arc $\gamma \subset c$ has combinatorial length $\pi / 3$, it must have an inward extension whose area comes from a single triangle $D$. $D$ cannot be of type $\mathrm{W}$, because white faces are meridional on a crossing circle cusp, and thus a triangle of this type, plus some bigons, cannot have their boundary segments add up to a progressive arc. Thus $D$ must be a triangle of type $\mathrm{S}$ or type $\mathrm{N}$.

Let $H$ be an inward extension of $c$. We claim that if $H$ contains a type- $\mathrm{N}$ triangle, then it consists entirely of type-N triangles. This is because by Corollary 5.7, a type$\mathrm{N}$ triangle $D$ cannot be glued to a type-S triangle or a bigon, and any other type of admissible disk glued to $D$ would contribute extra area and bring the total length above $n \pi / 3$. But if $H$ consists entirely of type- $\mathrm{N}$ triangles, $c$ consists entirely of longitudinal segments and never travels in the $\boldsymbol{w}$ direction. Thus we can conclude that $H$ cannot contain any type- $\mathrm{N}$ triangles.

The only remaining possibility is that $H$ consists entirely of type-S triangles and bigons. But in this case, all of $H$ is parallel to a single shaded disk, and again $c$ never traverses the lattice in the $\boldsymbol{w}$ direction. Thus the assumption that $\ell(c)=n \pi / 3$ leads to a contradiction.

We are now in a position to prove the theorems listed in the introduction.

Theorem 1.4. Let $K \subset S^{3}$ be a link with a prime, twist-reduced diagram $D(K)$. If $D(K)$ has at least two twist regions and every twist region of $D(K)$ contains at least 6 crossings, then $K$ is hyperbolic.

Proof. The assumption that $D(K)$ has at least two twist regions ensures that the constructions and results of Section 2 apply. Thus, by Theorem 2.4, $K$ is obtained by Dehn surgery on the crossing circles of a hyperbolic link $J$. By Proposition 5.13, every surgery slope $s_{i}$ on a crossing circle $C_{i}$ has combinatorial length $\ell\left(s_{i}\right)>2 \pi$. Therefore, by Theorem 4.15, $E(K)$ is hyperbolic. 
Theorem 1.7. Let $K$ be a link in $S^{3}$ with a prime, twist-reduced diagram $D(K)$. Suppose every twist region of $D(K)$ contains at least 6 crossings and each component of $K$ passes through at least 7 twist regions (counted with multiplicity). Then

(1) any non-trivial Dehn filling of some but not all components of $K$ is hyperbolic, and

(2) any non-trivial Dehn filling of all the components of $K$ is hyperbolike.

Proof. By Corollary 5.12, any non-trivial slope $s$ on a component of $K$ will have $\ell(s)>2 \pi$, and by Proposition 5.13, the same is true for surgery slopes on the crossing circles. Thus all surgery slopes on $\partial E(J)$ are sufficiently long. Conclusion (1) now follows by Theorem 4.15, and conclusion (2) by Theorem 4.14.

Theorem 1.5. Let $K \subset S^{3}$ be a link of $k$ components with a prime, twist-reduced diagram $D(K)$. If $D(K)$ has $t \geq 2$ twist regions and at least 6 crossings in each twist region, then

$$
\operatorname{genus}(K) \geq\left\lceil 1+\frac{t}{6}-\frac{k}{2}\right\rceil,
$$

where $\lceil\cdot\rceil$ is the ceiling function that rounds up to the nearest integer.

Proof. Let $F$ be a Seifert surface for $K$, that is, an orientable incompressible surface whose boundary is $K$. Then $F$ contains a punctured surface $G \subset E(J)$, where $\partial G$ consists of curves $\gamma_{1}, \ldots, \gamma_{k}$ that are longitudes of $K$ and curves $\gamma_{k+1}, \ldots, \gamma_{k+n}$ along the crossing circles. We can place $G$ in normal form in the polyhedra $P_{1}$ and $P_{2}$ and compute its combinatorial area. Observe that, by Corollary 5.12, the total length of $\gamma_{1}, \ldots, \gamma_{k}$ is at least $2 t \pi / 3$, because $K$ passes through each twist region twice. By Proposition 5.13, $\ell\left(\gamma_{i}\right)>2 \pi$ for $i>k$. Thus we can compute that

$$
\begin{aligned}
2 \pi \cdot \operatorname{genus}(F) & =2 \pi \cdot \operatorname{genus}(G) \\
& =2 \pi\left(1-\frac{1}{2} \chi(G)-\frac{1}{2}(k+n)\right) \\
& =2 \pi+\frac{1}{2} a(G)-\pi k-\pi n \\
& \geq 2 \pi+\frac{1}{2} \sum_{i=1}^{k} \ell\left(\gamma_{i}\right)-\pi k+\frac{1}{2} \sum_{i=k+1}^{k+n} \ell\left(\gamma_{i}\right)-\pi n \\
& \geq 2 \pi+\frac{t \pi}{3}-\pi k \\
& =2 \pi\left(1+\frac{t}{6}-\frac{k}{2}\right) .
\end{aligned}
$$

Since the genus of $F$ is an integer, we are done. 
Observe that the inequality in the computation is an equality whenever $G$ does not meet any crossing circles and consists of only ideal triangles. This can happen when the twist regions of $D(K)$ always meet in threes and $G$ lies in the projection plane. In this situation, Theorem 1.5 actually gives the exact value for the genus of $K$.

\section{References}

[1] Colin C. Adams, Augmented alternating link complements are hyperbolic. In Lowdimensional topology and Kleinian groups (Coventry/Durham, 1984), London Math. Soc. Lecture Note Ser. 112, Cambridge University Press, Cambridge 1986, 115-130. Zbl 0632.57008 MR 0903861

[2] Ian Agol, Bounds on exceptional Dehn filling. Geom. Topol. 4 (2000), 431-449 (electronic). Zbl 0959.57009 MR 1799796

[3] Kenneth Baker, Knots on Once-Punctured Torus Fibers. PhD thesis, University of Texas at Austin, 2004.

[4] Steven A. Bleiler and Craig D. Hodgson, Spherical space forms and Dehn filling. Topology 35 (3) (1996), 809-833. Zbl 0863.57009 MR 1396779

[5] Richard Crowell, Genus of alternating link types. Ann. of Math. (2) 69 (1959), 258-275. Zbl 0111.35803 MR 0099665

[6] Mario Eudave-Muñoz, On hyperbolic knots with Seifert fibered Dehn surgeries. Topology Appl. 121 (1-2) (2002), 119-141. Zbl 1009.57010 MR 1903687

[7] Mario Eudave-Muñoz and John Luecke, Knots with bounded cusp volume yet large tunnel number. J. Knot Theory Ramifications 8 (4) (1999), 437-446. Zbl 0942.57005 MR 1697382

[8] David Futer, Efstratia Kalfagianni, and Jessica S. Purcell, Dehn filling, volume, and the Jones polynomial. Preprint, 2006. arXiv:math.GT/0612138

[9] David Gabai, Genera of the arborescent links. Mem. Amer. Math. Soc. 59 (339) (1986), 1-98. Zbl 0585.57003 MR 0823442

[10] Wolfgang Haken, Theorie der Normalflächen. Acta Math. 105 (1961), 245-375. Zbl 0100.19402 MR 0141106

[11] Craig D. Hodgson and Steven P. Kerckhoff, Universal bounds for hyperbolic Dehn surgery. Ann. of Math. (2) 162 (1) (2005), 367-421. Zbl 05011497 MR 2178964

[12] Marc Lackenby, Word hyperbolic Dehn surgery. Invent. Math. 140 (2) (2000), 243-282. Zbl 0947.57016 MR 1756996

[13] Marc Lackenby, The canonical decomposition of once-punctured torus bundles. Comment. Math. Helv. 78 (2) (2003), 363-384. Zbl 1035.57010 MR 1988201

[14] Marc Lackenby, The volume of hyperbolic alternating link complements. With an appendix by Ian Agol and Dylan Thurston. Proc. London Math. Soc. (3) 88 (1) (2004), 204-224. Zbl 1041.57002 MR 2018964

[15] Marc Lackenby, Classification of alternating knots with tunnel number one. Comm. Anal. Geom. 13 (1) (2005), 151-185. Zbl 1084.57007 MR 2154670 
[16] William Menasco, Closed incompressible surfaces in alternating knot and link complements. Topology 23 (1) (1984), 37-44. Zbl 0525.57003 MR 0721450

[17] Kunio Murasugi, On the genus of the alternating knot. I, II. J. Math. Soc. Japan 10 (1958), 94-105, 235-248. Zbl 0106.16701 MR 0099664

[18] Grisha Perelman, The entropy formula for the Ricci flow and its geometric applications. Preprint, 2002.arXiv:math.DG/0211159

[19] Grisha Perelman, Ricci flow with surgery on three-manifolds. Preprint, 2003. arXiv:math.DG/0303109

[20] Jessica S. Purcell, Cusp Shapes of Hyperbolic Link Complements and Dehn Filling. PhD thesis, Stanford University, 2004.

[21] Igor Rivin, On geometry of convex ideal polyhedra in hyperbolic 3-space. Topology 32 (1) (1993), 87-92. Zbl 0784.52014 MR 1204408

[22] Eric Schoenfeld, Augmentations of Knot and Link Complements. Undergraduate thesis, Williams College, 2003.

[23] William P. Thurston, The geometry and topology of three-manifolds. Princeton Univ. Math. Dept. Notes, 1980. Available at http://www.msri.org/publications/books/gt3m/

[24] William P. Thurston, Three-dimensional manifolds, Kleinian groups and hyperbolic geometry. Bull. Amer. Math. Soc. (N.S.) 6 (3) (1982), 357-381. Zbl 0496.57005 MR 0648524

[25] William P. Thurston, Three-dimensional geometry and topology. Vol. 1, Princeton Math. Ser. 35, Princeton University Press, Princeton, NJ, 1997. Zbl 0873.57001 MR 1435975

[26] Ying-Qing Wu, Dehn surgery on arborescent knots. J. Differential Geom. 43 (1) (1996), 171-197. Zbl 0851.57018 MR 1424423

[27] Ying-Qing Wu, The classification of toroidal Dehn surgeries on Montesinos knots. Preprint, 2004. Available at http://www.math.uiowa.edu/ wu/papers/papers.html

Received June 14, 2005

David Futer, Mathematics Department, Michigan State University, East Lansing, MI 48824, U.S.A.

E-mail: dfuter@math.msu.edu

Jessica S. Purcell, Department of Mathematics, 1 University Station C1200, University of Texas at Austin, Austin, TX 78712, U.S.A.

E-mail: jpurcell@math.utexas.edu 